\title{
Shape optimization problems with Robin conditions on the free boundary
}

\author{
Dorin Bucur ${ }^{\mathrm{a}, 1}$, Alessandro Giacomini ${ }^{\mathrm{b}, *, 2}$ \\ a Laboratoire de Mathématiques, CNRS UMR 5127 Université de Savoie \& Institut Universitaire de France, Campus Scientifique, \\ 73376 Le-Bourget-Du-Lac, France \\ b DICATAM, Sezione di Matematica, Università degli Studi di Brescia, Via Valotti 9, 25133 Brescia, Italy
}

Received 13 January 2015; accepted 19 July 2015

Available online 31 August 2015

\begin{abstract}
We provide a free discontinuity approach to a class of shape optimization problems involving Robin conditions on the free boundary. More precisely, we identify a large family of domains on which such problems are well posed in a way that the extended problem can be considered a relaxed version of the corresponding one on regular domains, we prove existence of a solution and obtain some qualitative information on the optimal sets.
\end{abstract}

๑ 2015 L'Association Publications de l'Institut Henri Poincaré. Published by Elsevier B.V. All rights reserved.

Keywords: Free boundary problems; Shape optimization problems; Robin boundary conditions; Free discontinuity problems; Functions of bounded variation

\section{Introduction}

Let $D \subseteq \mathbb{R}^{d}$ be a design region which we assume to be open, bounded and with a Lipschitz boundary. Consider $B \subset D$ open and with a $C^{1}$-boundary and $g \in C^{1}\left(\mathbb{R}^{d}\right)$ such that

$$
0<c_{1} \leq g \leq c_{2} \quad \text { on } B .
$$

The main concern of the paper is the following shape optimization problem.

\footnotetext{
* Corresponding author.

E-mail addresses: dorin.bucur@univ-savoie.fr (D. Bucur), alessandro.giacomini@unibs.it (A. Giacomini).

1 The work of D.B. was supported by the ANR-12-BS01-0014-01 Geometrya.

2 The work of A.G. was supported by the Italian Ministry of Education, University and Research under the project "Calculus of Variations" (PRIN 2010-11). He is also member of the Gruppo Nazionale per L'Analisi Matematica, la Probabilità e loro Applicazioni (GNAMPA) of the Istituto Nazionale di Alta Matematica (INdAM).
} 
(P) Find $\Omega$ with Lipschitz boundary such that $B \subseteq \Omega \subseteq D$ and which minimizes the shape functional

$$
J(\Omega):=\min _{\substack{u \in W^{1, p}(\Omega) \\ u=g \text { on } B}}\left[\int_{\Omega} f(x, \nabla u) d x+\int_{\partial \Omega} \beta(x)|u|^{p} d \mathcal{H}^{d-1}+\gamma|\Omega|\right],
$$

where $p>1, \gamma \geq 0, f: \mathbb{R}^{d} \times \mathbb{R}^{d} \rightarrow[0,+\infty$ [ is continuous, with $\xi \mapsto f(x, \xi)$ convex and positively $p$-homogeneous, and $\beta: \mathbb{R}^{d} \rightarrow\left[0,+\infty\left[\right.\right.$ is continuous ( $\mathcal{H}^{d-1}$ stands for the $(d-1)$-dimensional Hausdorff measure).

The problem amounts to the determination of the "free boundary" $\partial \Omega$ of the optimal domain $\Omega$ on which the associated state function $u$ (which realizes $J(\Omega)$ ) satisfies a boundary condition of Robin type. In the case $p=2$, $f(x, \xi)=|\xi|^{2}$ and $\beta(x)=\beta$, the condition reduces precisely to the classical Robin condition

$$
\frac{\partial u}{\partial n}+\beta u=0 \quad \text { on } \partial \Omega
$$

where $n$ denotes the unit external normal.

The function $u$ satisfies also extra conditions on $\partial \Omega$ coming from optimality. Those new conditions, are referred to be overdetermined, but they do not play a fundamental role in our approach to the minimization problem.

In the two dimensional case with $f(x, \xi)=A(x) \xi \cdot \xi, \gamma=0$ and $p=2$, the problem can be interpreted as that of finding the shape of the membrane with minimal total energy among those with elastic properties given by the elastic moduli $A(x)$, prescribed transversal displacement $g$ on the part $B$, which are elastically supported at the boundary (with elastic forces with constant $\beta(x)$ ).

The existence of optimal domains for problem $(\mathrm{P})$ is unclear. In general, there are very few results in shape optimization where the existence of an optimal domain can be proved in a "natural" way, i.e. without imposing extra restrictive conditions, and the most of them hold for Dirichlet boundary conditions. For Robin b.c., the only analysis carried to understand existence concerns the first eigenvalue of the Robin Laplacian. Contrary to Dirichlet b.c., the general relaxed form of a Robin problem (i.e., a precise description of the limit of a sequence of Robin problems on a sequence of arbitrary, non-smooth, non-uniform domains) is not known. In this paper, we analyze a class of energy type functionals generalizing the Bernoulli free boundary problem in a nonlinear framework complemented by elastic boundary conditions.

The main result of our paper consists in the identification of a class of admissible domains $\mathcal{A}_{B}(D)$ containing the Lipschitz ones on which the minimization of $J$ can be carried out and can be considered as a relaxation of the original problem.

The class $\mathcal{A}_{B}(D)$ and the extension of $J$ to such a class is suggested by the study of the following free discontinuity functional

$$
F(u):=\int_{\mathbb{R}^{d}} f(x, \nabla u) d x+\int_{J_{u}} \beta(x)\left[\left(u^{+}\right)^{p}+\left(u^{-}\right)^{p}\right] d \mathcal{H}^{d-1}+\gamma|\{u>0\}|
$$

on the set of functions

$$
\mathcal{F}_{B, g}(D):=\left\{u \in S B V\left(\mathbb{R}^{d}\right): \operatorname{supp}(u) \subseteq \bar{D}, u \geq 0, u=g \text { on } B\right\} .
$$

Here $S B V$ denotes the space of special functions of bounded variation introduced by De Giorgi and Ambrosio [9] to deal with image segmentation problems. The link between $J$ and $F$ is obtained easily noticing that if $u$ is the state function of the regular domain $\Omega$ (which we can assume positive), then the extension of $u$ to $\mathbb{R}^{d}$ by zero outside $\Omega$ yields an element $\tilde{u}$ of $\mathcal{F}_{B, g}(D)$ such that

$$
F(\tilde{u})=\int_{\Omega} f(x, \nabla u) d x+\int_{\partial \Omega} \beta(x)|u|^{p} d \mathcal{H}^{d-1}+\gamma|\Omega|=J(\Omega) .
$$

The surface energy in (1.1) is rather unusual, involving the sum of the $p$-power of the traces of $u$. Its form, among the many yielding equality (1.2), is suggested by lower semicontinuity issues for the functional $F$.

We expect that the minimization problem

$$
\min _{u \in \mathcal{F}_{B, g}(D)} F(u)
$$


should provide information for the shape optimization problem $(\mathrm{P})$, in particular we expect optimal domains being given by the supports of optimal functions. Notice that this approach through a problem on functions is similar to that employed by Alt and Caffarelli in their pioneering paper [1], where a free boundary problem under Dirichlet conditions was studied. So the free discontinuity problem (1.3) is a sort of SBV-counterpart in the context of Robin boundary conditions of the problem considered in [1].

Clearly the connection between the free discontinuity problem (1.3) and problem $(\mathrm{P})$ is subordinated to the regularity properties of the optimal functions. We proceed as follows.

First of all, we show that the minimum problem (1.3) is well posed. This is not trivial because the compactness properties available for minimizing sequences are not compatible with Ambrosio's theorem in $S B V$ (see Theorem 2.1). Using standard results in SBV, one shows the existence of a candidate minimizer $u$ such that $u \geq 0$ and $u^{p} \in S B V\left(\mathbb{R}^{d}\right) \cap L^{\infty}\left(\mathbb{R}^{d}\right)$. The $S B V$-regularity is a consequence of optimality and relies on a bound from below on the support of its positivity set (see Theorem 3.5 and [6])

$$
u \geq \alpha>0 \quad \text { a.e. on }\{u>0\} .
$$

Such a property is immediately available in the classical context thanks to the Hopf Lemma and the Robin boundary condition (see Remark 3.8). In the context of the free discontinuity problems it is a sort of non-degeneracy property, coming from optimality.

In view of the bound from below (1.4) and that from above (given by $\|g\|_{\infty}$ ), the first two terms in the free discontinuity functional $F$ turn out to be estimated from above and below on the minimizer $u$ by the Mumford-Shah type functional

$$
M S(u):=\int_{\mathbb{R}^{d}} f(x, \nabla u) d x+\mathcal{H}^{d-1}\left(J_{u}\right)
$$

In particular the minimality property of $u$ for $F$ entails that (a suitable multiple of) $u$ is an almost-quasi minimizer of the functional MS (see Proposition 3.11). Such local minimizers are defined as follows: there exist $\Lambda \geq 1, \alpha>0$, $c_{\alpha}>0$ such that for every $B_{\rho}(x) \subset \subset D, v \in S B V_{l o c}(D)$ with $v=g$ on $B$ and $\{v \neq u\} \subseteq B_{\rho}(x)$

$$
\int_{B_{\rho}(x)} f(x, \nabla u) d x+\mathcal{H}^{d-1}\left(J_{u} \cap \bar{B}_{\rho}(x)\right) \leq \int_{B_{\rho}(x)} f(x, \nabla v) d x+\Lambda \mathcal{H}^{d-1}\left(J_{v} \cap \bar{B}_{\rho}(x)\right)+c_{\alpha} \rho^{d-1+\alpha} .
$$

This minimality property is weaker than that considered by De Giorgi, Carriero and Leaci in [10] since it can be $\Lambda>1$. Under suitable assumptions on $f$, we show (Theorem 2.3) that this weaker minimality still yields the essential closedness of $J_{u}$ in $D$, i.e.,

$$
\mathcal{H}^{d-1}\left(\left(\overline{J_{u}} \backslash J_{u}\right) \cap D\right)=0 .
$$

In the case $f(x, \xi)=|\xi|^{2}$, under certain supplementary hypotheses and without boundary condition (associated to $g$ ), such a result was proved by Siaudeau [13] following the lines of [10]. More recently, the same result has been proved in [6] on the basis of a monotonicity formula for the Mumford-Shah functional. Here we extend the analysis [10] to cover more general energy densities $f$ (for which monotonicity is unclear) and to treat boundary data (as in the case of [7]). A monotonicity formula similar to [6] can not hold for arbitrary $f$, but for some particular cases as $f(x, \xi)=|\xi|^{p}$, with $p \in(1,2)$ this can not be trivially excluded. Such a formula relies on a precise estimate from below of the ratio

$$
\inf _{u \in W^{1, p}\left(\partial B_{R}\right)} \frac{R \int_{\partial B_{R}}|\nabla u|^{p} d \mathcal{H}^{d-1}}{\int_{B_{R}}|\nabla u|^{p} d x},
$$

for $p \in(1,2)$ and $u$ satisfying $\Delta_{p} u=0$, which, up to our knowledge, is not known.

The analysis of problem (1.3) thus shows that the support of minimizers of $F$ is given by the connected component of $D \backslash \overline{J_{u}}$ on which $u$ does not vanish. This set belongs to the family

$$
\mathcal{A}_{B}(D):=\left\{\Omega \text { open }: B \subseteq \Omega \subseteq D, \partial \Omega \text { is } \mathcal{H}^{d-1} \text {-countably rectifiable with } \mathcal{H}^{d-1}(\partial \Omega)<+\infty\right\},
$$


which turns out to be the class we are looking for. Indeed we prove in Theorem 3.2 that

$$
\min _{\Omega \in \mathcal{A}_{B}(D)} J(\Omega),
$$

where the extension of $J$ to irregular domains is given in (3.3) below and is suggested by the free discontinuity functional $F$, is well posed and satisfies

$$
\min _{\Omega \in \mathcal{A}_{B}(D)} J(\Omega)=\inf _{\Omega \text { Lipschitz }} J(\Omega),
$$

i.e., the extended problem is a relaxed version of the original one. This last property is a consequence of density results in the sense of Cortesani and Toader [8] for the functional $F$ in $S B V$ (see Proposition 3.12).

The regularity of domains in $\mathcal{A}_{B}(D)$ is in the weak sense of geometric measure theory: in particular they are open sets with finite perimeter and they can admit in principle inner cracks. It is our opinion that the class

$\left\{\Omega\right.$ open and bounded in $\mathbb{R}^{d}: \partial \Omega$ is $\mathcal{H}^{d-1}$-countably rectifiable with $\left.\mathcal{H}^{d-1}(\partial \Omega)<+\infty\right\}$,

provides a natural framework for shape optimization problems under Robin conditions. In [5] we employed it to deal with the Faber-Krahn inequality of the first eigenvalue and associated semilinear variants (including the case of the torsional rigidity) of the Robin-Laplacian: the existence of an optimal domain in the class above permits to use geometrical arguments typical of shape optimization problems to show that minimizers are balls.

The paper is organized as follows. In Section 2 we fix the notation employed throughout the paper, and recall some basic facts concerning $S B V$-functions and free discontinuity problems. The main problem and the associated analysis outlined above is detailed in Section 3, while Section 4 contains the proof of the essential closedness of the jump sets of almost-quasi minimizers of (1.5).

\section{Notation and preliminaries}

Throughout the paper, $B_{r}(x)$ will denote the open ball of center $x \in \mathbb{R}^{d}$ and radius $r>0$. We will write $B_{r}$ in place of $B_{r}(0)$. We say that $A \subset \subset B$ if $\bar{A}$ is compact and contained in $B$. If $E \subset \mathbb{R}^{d}$, we will denote its volume by $|E|$, and $1_{E}$ will stand for its characteristic function, i.e., $1_{E}(x)=1$ if $x \in E$ and $1_{E}(x)=0$ if $x \notin E$. We set $\omega_{d}:=\left|B_{1}\right|$.

For $A \subseteq \mathbb{R}^{d}$ open set and $p \geq 1, L^{p}(A)$ will denote the usual Lebesgue space of $p$-summable functions, while $W^{1, p}(A)$ will denote the Sobolev space of functions in $L^{p}(A)$ whose derivative in the sense of distributions is $p$-summable. Moreover $\|u\|_{\infty}$ will stand for the sup-norm of $u$, while $\operatorname{supp}(u)$ will denote the set $\{u \neq 0\}$, well defined up to zero Lebesgue measure. We will say that $\{u \neq v\} \subseteq B_{\rho}(x)$ if $u=v$ a.e. outside $B_{\rho}(x)$.

Finally we will use the following notation: for $a, b \in \mathbb{R}$

$$
a \wedge b:=\min \{a, b\} \quad \text { and } \quad a \vee b:=\max \{a, b\} .
$$

\subsection{Functions of bounded variation}

Let $A \subseteq \mathbb{R}^{d}$ be an open set. We say that $u \in B V(A)$ if $u \in L^{1}(A)$ and its derivative in the sense of distributions is a finite Radon measure on $A$, i.e., $D u \in \mathcal{M}_{b}\left(A ; \mathbb{R}^{d}\right) . B V(A)$ is called the space of functions of bounded variation on $A$. $B V(A)$ is a Banach space under the norm $\|u\|_{B V(A)}:=\|u\|_{L^{1}(A)}+\|D u\|_{\mathcal{M}_{b}\left(A ; \mathbb{R}^{d}\right)}$. We refer the reader to [2] for an exhaustive treatment of the space $B V$.

Concerning the fine properties, a function $u \in B V(A)$ (or better every representative of $u$ ) is a.e. approximately differentiable on $A$, with approximate gradient $\nabla u \in L^{1}\left(A ; \mathbb{R}^{d}\right)$. Moreover, the jump set $J_{u}$ is a $\mathcal{H}^{d-1}$-countably rectifiable set, i.e., $J_{u} \subseteq \cup_{i \in \mathbb{N}} M_{i}$ up to a $\mathcal{H}^{d-1}$-negligible set, with $M_{i}$ a $C^{1}$-hypersurface in $\mathbb{R}^{d}$. The measure $D u$ admits the following representation for every Borel set $B \subseteq A$ :

$$
D u(B)=\int_{B} \nabla u d x+\int_{J_{u} \cap B}\left(u^{+}-u^{-}\right) v_{u} d \mathcal{H}^{d-1}+D^{c} u(B),
$$

where $v_{u}(x)$ is the normal to $J_{u}$ at $x$, and $D^{c} u$ is singular with respect to the Lebesgue measure and concentrated outside $J_{u} . D^{c} u$ is usually referred to as the Cantor part of $D u . u^{ \pm}$are the upper and lower approximate limits of $u$ at $x$. The normal $v_{u}$ coincides $\mathcal{H}^{d-1}$-a.e. on $J_{u}$ with the normal to the hypersurfaces $M_{i}$. The direction of $v_{u}(x)$ is 
chosen in such a way that $u^{ \pm}(x)$ is the approximate limit of $u$ at $x$ on the sets $\left\{y \in \mathbb{R}^{d}: v_{u}(x) \cdot(y-x) \gtrless 0\right\}$. Moreover, $u^{ \pm}$coincide $\mathcal{H}^{d-1}$-almost everywhere on $J_{u}$ with the traces $\gamma^{ \pm}(u)$ of $u$ on $J_{u}$ which are defined by the following Lebesgue-type limit quotient relation

$$
\lim _{r \rightarrow 0} \frac{1}{r^{d}} \int_{B_{r}^{ \pm}(x)}\left|u(x)-\gamma^{ \pm}(u)(x)\right| d x=0
$$

where $B_{r}^{ \pm}(x):=\left\{y \in B_{r}(x): v_{u}(x) \cdot(y-x) \gtrless 0\right\}$ (see [2, Remark 3.79]).

If $A$ is bounded and with a Lipschitz boundary, then $B V(A) \hookrightarrow L^{d / d-1}(A)$. Moreover, the following compactness result holds: if $\left(u_{n}\right)_{n \in \mathbb{N}}$ is bounded in $B V(A)$, there exist $u \in B V(A)$ and a subsequence $\left(u_{n_{k}}\right)_{k \in \mathbb{N}}$ such that

$$
u_{n_{k}} \rightarrow u \quad \text { strongly in } L^{1}(A)
$$

and

$$
D u_{n_{k}} \rightarrow D u \quad \text { weakly* in the sense of measures. }
$$

We say in this case that $u_{n_{k}} \stackrel{*}{\rightarrow} u$ weakly* in $B V(A)$.

We say that $u \in S B V(A)$ if $u \in B V(A)$ and $D^{c} u=0 . S B V(A)$ is called the space of special functions of bounded variation on $A$. This space is very useful in free discontinuity problems in view of the following compactness and lower-semicontinuity result due to L. Ambrosio (see [2, Theorems 4.7-4.8]).

Theorem 2.1. Let $A \subset \mathbb{R}^{d}$ be open and bounded, $\left.p \in\right] 1,+\infty\left[\right.$, and let $\left(u_{n}\right)_{n \in \mathbb{N}}$ be a sequence in $S B V(A)$ such that

$$
\sup _{n} \int_{A}\left|\nabla u_{n}\right|^{p} d x+\mathcal{H}^{d-1}\left(J_{u_{n}}\right)+\left\|u_{n}\right\|_{\infty}<+\infty .
$$

Then there exist $u \in S B V(A)$ with $\nabla u \in L^{p}\left(A ; \mathbb{R}^{d}\right)$ and a subsequence $\left(u_{n_{k}}\right)_{k \in \mathbb{N}}$ such that

$$
\begin{aligned}
& u_{n_{k}} \rightarrow u \quad \text { strongly in } L^{1}(A), \\
& \nabla u_{n_{k}} \rightarrow \nabla u \quad \text { weakly in } L^{p}\left(A ; \mathbb{R}^{d}\right)
\end{aligned}
$$

and

$$
\mathcal{H}^{d-1}\left(J_{u}\right) \leq \liminf _{n \rightarrow+\infty} \mathcal{H}^{d-1}\left(J_{u_{n}}\right) .
$$

In the following we will use the notation

$$
S B V^{p}(A):=\left\{u \in S B V(A): \nabla u \in L^{p}\left(A ; \mathbb{R}^{d}\right) \text { and } \mathcal{H}^{d-1}\left(J_{u}\right)<+\infty\right\} .
$$

\subsection{Regularity results for almost-quasi minimizers of free discontinuity problems}

Let $\Omega \subseteq \mathbb{R}^{d}$ be an open set, and let $\Omega^{\prime} \subseteq \mathbb{R}^{d}$ be open with $\Omega \subseteq \Omega^{\prime}$ and such that $\Omega^{\prime} \backslash \bar{\Omega}$ has a $C^{1}$-boundary. Let $g \in C^{1}\left(\mathbb{R}^{\bar{d}}\right)$. For $u \in S B V_{\text {loc }}\left(\Omega^{\prime}\right)$ with $u=\bar{g}$ on $\Omega^{\prime} \backslash \bar{\Omega}$, let us consider the free discontinuity functional

$$
F(u):=\int_{\Omega^{\prime}} f(x, \nabla u) d x+\mathcal{H}^{d-1}\left(J_{u}\right) .
$$

We assume that $f: \Omega^{\prime} \times \mathbb{R}^{d} \rightarrow[0,+\infty[$ satisfies the following assumptions.

$\left(H_{1}\right) f$ is continuous and there exist $L>0$ and $p>1$ such that for every $x \in \Omega^{\prime}, \xi \in \mathbb{R}^{d}$ and $t>0$

$$
f(x, t \xi)=t^{p} f(x, \xi), \quad L^{-1}|\xi|^{p} \leq f(x, \xi) \leq L|\xi|^{p} .
$$

$\left(H_{2}\right)$ For every $x \in \Omega^{\prime}$, the map $\xi \mapsto f(x, \xi)$ is convex. 
$\left(H_{3}\right)$ There exists $\mu>0$ such that for every $x_{0} \in \Omega^{\prime}, \varphi \in C_{c}^{1}\left(B_{1}\right)$ and $\xi \in \mathbb{R}^{d}$,

$$
\int_{B_{1}}\left[f\left(x_{0}, \xi+\nabla \varphi\right)-f\left(x_{0}, \xi\right)\right] d x \geq \mu \int_{B_{1}}\left(|\xi|^{2}+|\nabla \varphi|^{2}\right)^{\frac{p-2}{2}}|\nabla \varphi|^{2} d x .
$$

We are interested in the following notion of local minimality.

Definition 2.2 (Almost-quasi minimality). Let $u \in S B V_{l o c}^{p}\left(\Omega^{\prime}\right)$ be such that $u=g$ on $\Omega^{\prime} \backslash \bar{\Omega}$. We say that $u$ is an almost-quasi minimizer for the functional $F$ in (2.1) with boundary condition $g$ if there exist $\Lambda \geq 1, \alpha>0$ and $c_{\alpha}>0$ such that for every ball $B_{\rho}\left(x_{0}\right) \subset \subset \Omega^{\prime}$

$$
\int_{B_{\rho}\left(x_{0}\right)} f(x, \nabla u) d x+\mathcal{H}^{d-1}\left(J_{u} \cap \bar{B}_{\rho}\left(x_{0}\right)\right) \leq \int_{B_{\rho}\left(x_{0}\right)} f(x, \nabla v) d x+\Lambda \mathcal{H}^{d-1}\left(J_{v} \cap \bar{B}_{\rho}\left(x_{0}\right)\right)+c_{\alpha} \rho^{d-1+\alpha}
$$

for every $v \in S B V_{l o c}^{p}\left(\Omega^{\prime}\right), v=g$ on $\Omega^{\prime} \backslash \bar{\Omega}$, and such that $\{v \neq u\} \subseteq B_{\rho}\left(x_{0}\right)$.

The following result will be pivotal in our analysis. It states that the jump set of almost-quasi minimizers enjoys the Ahlfors regularity à la De Giorgi-Carriero-Leaci [10], i.e., it is essentially closed.

Theorem 2.3 (Essential closedness of the jump set). Let $\Omega \subseteq \Omega^{\prime} \subseteq \mathbb{R}^{d}$ be open and such that $\Omega^{\prime} \backslash \bar{\Omega}$ has a $C^{1}$-boundary. Let $g \in C^{1}\left(\mathbb{R}^{d}\right)$ and let $f: \Omega^{\prime} \times \mathbb{R}^{d} \rightarrow\left[0,+\infty\left[\right.\right.$ satisfy $\left(H_{1}\right)-\left(H_{3}\right)$. Let $u \in S B V_{\text {loc }}^{p}\left(\Omega^{\prime}\right)$ be an almostquasi minimizer for the functional (2.1) with boundary condition g according to Definition 2.2.

Given $\tilde{\Omega} \subset \subset \Omega^{\prime}$, there exist $\varepsilon_{0}>0$ and $\rho_{0}>0$ depending on $\tilde{\Omega}$ such that for every $x \in J_{u}, x \in \tilde{\Omega}$ and $\rho<\rho_{0}$

$$
\frac{1}{\varepsilon_{0}} \rho^{d-1} \geq \mathcal{H}^{d-1}\left(J_{u} \cap B_{\rho}(x)\right) \geq \varepsilon_{0} \rho^{d-1} .
$$

In particular

$$
\mathcal{H}^{d-1}\left(\left(\overline{J_{u}} \backslash J_{u}\right) \cap \Omega^{\prime}\right)=0,
$$

i.e., the jump set of $u$ is essentially closed in $\Omega^{\prime}$.

For $\Lambda=1, f=|\xi|^{2}$ and $\Omega^{\prime}=\Omega$, i.e., for quasi minimizers for the original Mumford-Shah functional, the previous property reduces to the celebrated of De Giorgi, Carriero and Leaci [10]. Their approach has then be adapted to cover more general energy densities: the case of $p$-homogeneous functions $f(\xi)$ can be found e.g. in [2, Chapter 7]. The case with boundary conditions has been dealt by Carriero and Leaci in [7].

Ahlfors regularity for $\Lambda>1$ without boundary conditions has been proved by Siaudeau in [13] along the lines of [10], but in the presence of some supplementary a priori hypotheses, and also in [6] on the basis of a general monotonicity formula for the Mumford-Shah functional.

We will give the proof of Theorem 2.3 in Section 4: the main difference with respect to [13] is that we consider a weaker setting with more general energy densities $f$ and we take into account boundary conditions, and so our contribution is essentially technical. The reader already acquainted with the ideas of [10] and of [7] could skip the proof of Theorem 2.3 without prejudice.

\subsection{A density result for free discontinuity functionals}

We will make use of a density result in $S B V$ due to Cortesani and Toader [8]. In order to formulate the statement, we will say that $u \in \operatorname{SBV}(\Omega)$ with $\Omega$ open set in $\mathbb{R}^{d}$ has polyhedral jumps if $\overline{J_{u}} \cap \Omega$ is the intersection with $\Omega$ of the union of a finite number of $(d-1)$-dimensional simplexes. The density result is the following (see [8, Theorem 3.1]).

Theorem 2.4. Let $\Omega \subseteq \mathbb{R}^{d}$ be open and with Lipschitz boundary, and let $p>1$. Let $u \in S B V(\Omega) \cap L^{\infty}(\Omega)$ be such that $\nabla u \in L^{p}\left(\Omega ; \mathbb{R}^{d}\right)$ and $\mathcal{H}^{d-1}\left(J_{u}\right)<+\infty$. 
There exists $\left(u_{n}\right)_{n \in \mathbb{N}}$ such that the following items hold true for every $n \in \mathbb{N}$.

(a) $\mathcal{H}^{d-1}\left(\left(\overline{J_{u_{n}}} \backslash J_{u_{n}}\right) \cap \Omega\right)=0$.

(b) $J_{u_{n}}$ is polyhedral in $\Omega$.

(c) $u_{n} \in W^{k, \infty}\left(\Omega \backslash \overline{u_{u_{n}}}\right)$ for every $k \geq 1$.

\section{Moreover}

$$
\begin{aligned}
& u_{n} \rightarrow u \quad \text { strongly in } L^{1}(\Omega), \\
& \nabla u_{n} \rightarrow \nabla u \quad \text { strongly in } L^{p}\left(\Omega ; \mathbb{R}^{d}\right),
\end{aligned}
$$

and

$$
\limsup _{n \rightarrow+\infty} \int_{J_{u_{n}} \cap A} \varphi\left(x, u_{n}^{+}, u_{n}^{-}, v_{u_{n}}\right) d \mathcal{H}^{d-1} \leq \int_{J_{u} \cap A} \varphi\left(x, u^{+}, u^{-}, v_{u}\right) d \mathcal{H}^{d-1}
$$

for every open set $A \subset \subset \Omega$ and every upper semicontinuous function $\varphi: \Omega \times \mathbb{R} \times \mathbb{R} \times S^{d-1} \rightarrow[0,+\infty[$ such that $\varphi(x, a, b, v)=\varphi(x, b, a,-v)$ for every $x \in \Omega, a, b \in \mathbb{R}$ and $v \in S^{d-1}$.

\section{A free boundary problem with Robin conditions}

With the notations of Section 2 , let $\Omega^{\prime}=\mathbb{R}^{d}$ and $f: \mathbb{R}^{d} \times \mathbb{R}^{d} \rightarrow\left[0,+\infty\left[\right.\right.$ satisfy the assumptions $\left(H_{1}\right),\left(H_{2}\right)$, $\left(H_{3}\right)$. Let $\beta: \mathbb{R}^{d} \rightarrow[0,+\infty[$ be a continuous function such that

$$
\forall x \in \mathbb{R}^{d}: 0<\beta_{1} \leq \beta(x) \leq \beta_{2}<+\infty
$$

for some positive constants $\beta_{1}, \beta_{2}$. Let $D \subseteq \mathbb{R}^{d}$ be a design region which we assume to be an open bounded set with a Lipschitz boundary. Consider $B \subset D$ open and with $C^{1}$-boundary, and $g \in C^{1}\left(\mathbb{R}^{d}\right)$ such that

$$
0<c_{1} \leq g \leq c_{2} \quad \text { on } B .
$$

For every domain $\Omega$ with Lipschitz boundary such that $B \subseteq \Omega \subseteq D$, let us consider the shape functional

$$
J(\Omega):=\min _{\substack{u \in W^{1, p}(\Omega) \\ u=g \text { on } B}}\left[\int_{\Omega} f(x, \nabla u) d x+\int_{\partial \Omega} \beta(x)|u|^{p} d \mathcal{H}^{d-1}\right]+\gamma|\Omega|,
$$

where $\gamma \in[0,+\infty[$.

We are interested in the minimization of $J$ among all admissible domains. Unfortunately, the existence of domains which minimize $J$ is unclear, due to the lack of compactness properties for minimizing sequences of Lipschitz sets.

In order to achieve the existence of optimal domains, following [5] we relax the previous problem to the family of sets

$$
\mathcal{A}_{B}(D):=\left\{\Omega \text { open }: B \subseteq \Omega \subseteq D, \partial \Omega \text { is } \mathcal{H}^{d-1} \text {-countably rectifiable with } \mathcal{H}^{d-1}(\partial \Omega)<+\infty\right\}
$$

by setting for $\Omega \in \mathcal{A}_{B}(D)$

$$
J(\Omega):=\min _{\substack{u \in W^{1, p}(\Omega) \cap L^{\infty}(\Omega) \\ u=g \text { on } B}}\left[\int_{\Omega} f(x, \nabla u) d x+\int_{\partial \Omega} \beta(x)\left[\left|u^{+}\right|^{p}+\left|u^{-}\right|^{p}\right] d \mathcal{H}^{d-1}\right]+\gamma|\Omega| .
$$

To make sense of the previous expression, notice that if we extend $u$ to zero outside $\Omega$, we obtain a function of bounded variation on $\mathbb{R}^{d}$ such that $J_{u} \subseteq \partial \Omega: u^{ \pm}$are thus the traces of $u$ on $\partial \Omega$ in the sense of $B V$-theory, which turn out to be well defined up to a $\mathcal{H}^{d-1}$-negligible set. Notice that we admit two traces since the geometry of $\Omega$ is compatible with the presence of inner cracks. If $\Omega$ is regular, then the previous expression for $J$ coincides clearly with the classical one. 
Remark 3.1. The existence of a minimizer $u$ in the definition (3.3) of $J$ such that

$$
0 \leq u \leq\|g\|_{\infty}
$$

is easily proved. Indeed, if $\left(u_{n}\right)_{n \in \mathbb{N}}$ is a minimizing sequence, by truncation we may assume $0 \leq u_{n} \leq\|g\|_{\infty}$. If $u \in W^{1, p}(\Omega)$ is a limit point of the sequence in the weak topology, then it is a minimizer since

$$
\int_{\Omega} f(x, \nabla u) d x \leq \liminf _{n \rightarrow+\infty} \int_{\Omega} f\left(x, \nabla u_{n}\right) d x
$$

and, using a standard slicing argument (see e.g. [5, Lemma 6.16])

$$
\int_{\partial \Omega} \beta(x)\left[\left|u^{+}\right|^{p}+\left|u^{-}\right|^{p}\right] d \mathcal{H}^{d-1} \leq \liminf _{n \rightarrow+\infty} \int_{\partial \Omega} \beta(x)\left[\left|u_{n}^{+}\right|^{p}+\left|u_{n}^{-}\right|^{p}\right] d \mathcal{H}^{d-1} .
$$

The main result of the paper is the following.

Theorem 3.2. Let $D \subseteq \mathbb{R}^{d}$ be an open, bounded set with Lipschitz boundary, let $f: \mathbb{R}^{d} \times \mathbb{R}^{d} \rightarrow[0,+\infty[$ satisfy $\left(H_{1}\right)-\left(H_{3}\right)$, and let $\beta: \mathbb{R}^{d} \rightarrow\left[0,+\infty\left[\right.\right.$ satisfy (3.1). Given $B \subset D$ open and with a $C^{1}$-boundary, and $g \in C^{1}\left(\mathbb{R}^{d}\right)$ satisfying (3.2), the shape optimization problem

$$
\min _{\Omega \in \mathcal{A}_{B}(D)} J(\Omega)
$$

admits a solution $\tilde{\Omega}$, which moreover satisfies

$$
J(\tilde{\Omega})=\inf _{\substack{\Omega \in \mathcal{A}_{B}(D) \\ \Omega \text { is Lipschitz }}} J(\Omega) .
$$

Theorem 3.2 thus proves that the new shape optimization problem (3.4) is well posed and it is a relaxed version of the original one on regular domains.

In order to address problem (3.4) we consider the free discontinuity functional

$$
F(u):=\int_{\mathbb{R}^{d}} f(x, \nabla u) d x+\int_{J_{u}} \beta(x)\left[\left(u^{+}\right)^{p}+\left(u^{-}\right)^{p} \mid\right] d \mathcal{H}^{d-1}+\gamma|\{u>0\}|
$$

on the set

$$
\mathcal{F}_{B, g}(D):=\left\{u \in S B V\left(\mathbb{R}^{d}\right): \operatorname{supp}(u) \subseteq \bar{D}, u \geq 0, u=g \text { on } B\right\} .
$$

The basic idea is that the minimization of $F$ defined on functions should be easier than that of $J$ defined on sets. Moreover we expect to recover an optimal domain by considering the support $\{u>0\}$ of an optimal function $u$. The key point for this to hold true is to show that minimizers of (3.6) are regular enough to guarantee that their support belongs to $\mathcal{A}_{B}(D)$.

Existence of minimizers for (3.6) on $\mathcal{F}_{B, g}(D)$ is by no means obvious, since coercivity properties of minimizing sequences are not compatible with the usual compactness in $S B V$ given by Ambrosio's theorem. Let indeed $\left(u_{n}\right)_{n \in \mathbb{N}}$ be a minimizing sequence. By truncation, is not restrictive to assume

$$
0 \leq u_{n} \leq\|g\|_{\infty} .
$$

Then we get by comparing with $g 1_{D}$

$$
\int_{D}\left|\nabla u_{n}\right|^{p} d x+\int_{u_{u_{n}}}\left|u_{n}^{+}\right|^{p}+\left|u_{n}^{-}\right|^{p} d \mathcal{H}^{d-1} \leq C,
$$

with $C$ independent of $n$. We thus see that no bounds on the $\mathcal{H}^{d-1}$-measure of the jump sets are available, so that a direct application of Ambrosio's theorem is forbidden. 
We have however the following compactness result.

Proposition 3.3. There exists $u \in L^{\infty}\left(\mathbb{R}^{d}\right)$ with $u \geq 0$, $\operatorname{supp}(u) \subseteq \bar{D}$ and $u^{p} \in S B V\left(\mathbb{R}^{d}\right)$ such that, up to a subsequence,

$$
u_{n} \rightarrow u \quad \text { a.e. in } \mathbb{R}^{d} .
$$

Moreover for every $v \in \mathcal{F}_{B, g}(D)$

$$
\int_{\mathbb{R}^{d}} f(x, \nabla u) d x+\int_{J_{u}} \beta(x)\left[\left(u^{+}\right)^{p}+\left(u^{-}\right)^{p}\right] d \mathcal{H}^{d-1}+\gamma|\{u>0\}| \leq F(v)
$$

(notice that $u$ is approximately differentiable a.e. since $u^{p} \in S B V\left(\mathbb{R}^{d}\right)$ ).

Proof. By the chain rule in $B V$ we get that $w_{n}:=u_{n}^{p} \in S B V\left(\mathbb{R}^{d}\right)$ with

$$
\nabla w_{n}=p u_{n}^{p-1} \nabla u_{n} .
$$

In view of (3.7) and since $u_{n} \leq\|g\|_{\infty}$ we obtain

$$
\sup _{n}\left|D w_{n}\right|\left(\mathbb{R}^{d}\right)<+\infty \text {. }
$$

Up to a subsequence we get

$$
w_{n} \rightarrow w \quad \text { strongly in } L^{1}\left(\mathbb{R}^{d}\right)
$$

for some $w \in B V\left(\mathbb{R}^{d}\right)$ with $w \geq 0$ and $\operatorname{supp}(w) \subseteq \bar{D}$. Notice that Ambrosio's theorem can be applied locally to $w_{n} \vee \varepsilon$ for every $\varepsilon>0$. We thus get $w \vee \varepsilon \in S B V_{l o c}\left(\mathbb{R}^{d}\right)$ for every $\varepsilon>0$, so that $w \in S B V\left(\mathbb{R}^{d}\right)$.

We set

$$
u:=w^{1 / p},
$$

so that the first part of the statement is proved.

Let us come to the minimality property of $u$. Notice that the sequence $\left(\left(u_{n}-\varepsilon\right)_{+}\right)_{n \in \mathbb{N}}$ satisfies the assumptions of Ambrosio's compactness theorem for every $\varepsilon>0$. By the lower semicontinuity for surface energies [4, Theorem 2.12] we get in view of the arbitrariness of $\varepsilon$

$$
\int_{J_{u}} \beta(x)\left[\left(u^{+}\right)^{p}+\left(u^{-}\right)^{p}\right] d \mathcal{H}^{d-1} \leq \liminf _{n \rightarrow+\infty} \int_{J_{u_{n}}} \beta(x)\left[\left(u_{n}^{+}\right)^{p}+\left(u_{n}^{-}\right)^{p}\right] d \mathcal{H}^{d-1} .
$$

If up to a subsequence

$$
\nabla u_{n} \rightarrow \Phi \quad \text { weakly in } L^{p}\left(\mathbb{R}^{d} ; \mathbb{R}^{d}\right)
$$

we deduce from (3.8) and the equality $\nabla\left(u_{n}-\varepsilon\right)_{+}=\nabla u_{n} 1_{\left\{u_{n} \geq \varepsilon\right\}}$ that

$$
\Phi=\nabla u \quad \text { a.e. on }\{u>0\} .
$$

By lower semicontinuity we have

$$
\int_{\mathbb{R}^{d}} f(x, \nabla u) d x \leq \int_{\mathbb{R}^{d}} f(x, \Phi) d x \leq \liminf _{n \rightarrow+\infty} \int_{\mathbb{R}^{d}} f\left(x, \nabla u_{n}\right) d x .
$$

Since we also have

$$
|\{u>0\}| \leq \liminf _{n \rightarrow \infty}\left|\left\{u_{n}>0\right\}\right|,
$$

the minimality follows collecting (3.9) and (3.10).

The function provided by the previous proposition does not a priori belong to $\mathcal{F}_{B, g}(D)$ since the $S B V$-regularity is unclear. In order to address such an issue, we introduce the following notion of subsolution. 
Definition 3.4 (Subsolution). We say that $u \in \mathcal{F}_{B, g}(D)$ is a subsolution for the functional

$$
u \mapsto \int_{\mathbb{R}^{d}} f(x, \nabla u) d x+\int_{J_{u}} \beta(x)\left[\left(u^{+}\right)^{p}+\left(u^{-}\right)^{p}\right] d \mathcal{H}^{d-1}
$$

if for every $v \in \mathcal{F}_{B, g}(D)$ with $0 \leq v \leq u$ we have

$$
F(u) \leq F(v) \text {. }
$$

Clearly, for every $\gamma \geq 0$ any minimizer given by Proposition 3.3 is also a subsolution in the sense defined above. The following non-degeneracy result for subsolutions, which is related to the Hopf principle (see Remark 3.8), holds true.

Theorem 3.5 (Bound from below). Let $u$ be a subsolution for (3.11). Then there exists $\alpha>0$ such that

$$
u \geq \alpha \quad \text { a.e. on }\{u>0\} .
$$

Proof. Let $\varepsilon<c_{1}$ (defined in (3.2)) be such that $u 1_{\{u>\varepsilon\}} \in S B V\left(\mathbb{R}^{d}\right)$. Then such a function belongs to $\mathcal{F}_{B, g}(D)$, so that, by comparison with $u$

$$
\begin{aligned}
& \int_{\mathbb{R}^{d}} f(x, \nabla u) d x+\int_{J_{u}} \beta(x)\left[\left(u^{+}\right)^{p}+\left(u^{-}\right)^{p}\right] d \mathcal{H}^{d-1} \\
& \leq \int_{\{u>\varepsilon\}} f(x, \nabla u) d x+\int_{\left\{u^{-}<\varepsilon \leq u^{+}\right\} \cap J_{u}} \beta(x)\left(u^{+}\right)^{p} d \mathcal{H}^{d-1}+ \\
& \quad+\int_{\left\{\varepsilon \leq u^{-}<u^{+}\right\} \cap J_{u}} \beta(x)\left[\left(u^{+}\right)^{p}+\left(u^{-}\right)^{p}\right] d \mathcal{H}^{d-1}+\int_{\partial^{e}\{u>\varepsilon\} \backslash J_{u}} \beta(x) \varepsilon^{p} d \mathcal{H}^{d-1}
\end{aligned}
$$

where $\partial^{e}\{u>\varepsilon\}$ stands for the essential boundary of the set $\{u>\varepsilon\}$, i.e., the points with density neither zero nor one (see [2, Definition 3.60]).

By the assumptions on $f$ and $\beta$ we deduce

$$
L^{-1} \int_{\{u \leq \varepsilon\}}|\nabla u|^{p} d x+\beta_{1} \int_{\left\{u^{-}<u^{+} \leq \varepsilon\right\} \cap J_{u}}\left[\left(u^{-}\right)^{p}+\left(u^{+}\right)^{p}\right] d \mathcal{H}^{d-1} \leq \beta_{2} \varepsilon^{p} \mathcal{H}^{d-1}\left(\partial^{e}\{u>\varepsilon\} \backslash J_{u}\right)
$$

which entails for a.e. $0<\delta<\varepsilon$

$$
\int_{\{u \leq \varepsilon\}}|\nabla u|^{p} d x+L \beta_{1} \delta^{p} \mathcal{H}^{d-1}\left(\partial^{e}\{\delta<u<\varepsilon\} \cap J_{u}\right) \leq L \beta_{2} \varepsilon^{p} \mathcal{H}^{d-1}\left(\partial^{e}\{u>\varepsilon\} \backslash J_{u}\right) .
$$

Setting

$$
\begin{aligned}
& E(\varepsilon):=\int_{\{u<\varepsilon\}}|\nabla u|^{p} d x, \quad \gamma(\delta, \varepsilon):=\mathcal{H}^{d-1}\left(\partial^{e}\{\delta<u<\varepsilon\} \cap J_{u}\right), \\
& \quad \text { and } \quad h(\varepsilon):=\mathcal{H}^{d-1}\left(\partial^{e}\{u \geq \varepsilon\} \backslash J_{u}\right),
\end{aligned}
$$

we deduce thus that for a.e. $0<\delta<\varepsilon<c_{1}$

$$
E(\varepsilon)+L \beta_{1} \delta^{p} \gamma(\delta, \varepsilon) \leq L \beta_{2} \varepsilon^{p} h(\varepsilon) .
$$

Let $\eta>0$ and set

$$
\varepsilon_{i}:=\frac{5}{6} \eta+\frac{2^{-i}}{6} \eta \quad \text { and } \quad \delta_{i}:=\frac{2}{3} \eta-\frac{2^{-i}}{6} \eta .
$$


We have for $i \rightarrow+\infty$

$$
\varepsilon_{i} \rightarrow e_{\infty}:=\frac{5}{6} \eta \quad \text { and } \quad \delta_{i} \rightarrow \delta_{\infty}:=\frac{2}{3} \eta .
$$

Setting

$$
\Omega(\delta, \varepsilon):=\{\delta<u<\varepsilon\},
$$

we will see that there exists $\eta_{0}>0$ small enough such that for $\eta<\eta_{0}$

$$
\left|\Omega\left(\frac{2}{3} \eta, \frac{5}{6} \eta\right)\right| \int_{\frac{2}{3} \eta}^{\frac{5}{6} \eta} h(s) d s=0 .
$$

Since in view of the isoperimetric inequality we have

$$
|\Omega(\delta, \varepsilon)|^{\frac{d-1}{d}} \leq C_{d}(h(\varepsilon)+h(\delta)+\gamma(\delta, \varepsilon)),
$$

relation (3.17) together with (3.14) entails that

$$
\left|\Omega\left(\frac{2}{3} \eta, \frac{5}{6} \eta\right)\right|=0 \text {. }
$$

Since $\eta<\eta_{0}$ is arbitrary, we get that

$$
u \geq \frac{5}{6} \eta_{0} \quad \text { a.e. on } \operatorname{supp}(u) .
$$

The proof of (3.17) will be the outcome of an iteration scheme which resembles under certain aspects the iteration à la De Giorgi in the study of elliptic regularity. We divide the proof in several steps.

Step 1: The main inequalities. Let us set for $i \in \mathbb{N}$

$$
a_{i}:=\int_{\delta_{i}}^{\varepsilon_{i}} h(s) d s \quad \text { and } \quad b_{i}:=\left|\Omega\left(\delta_{i}, \varepsilon_{i}\right)\right|,
$$

where $h$ is defined in (3.13), while $\Omega(\delta, \varepsilon)$ is given in (3.16).

We claim that there exist $c_{1}, c_{2}>0$ depending only on $d, p$ and $\beta$ such that for every $i \geq 1$

$$
\left\{\begin{array}{l}
a_{i} \leq c_{1} 2^{i} b_{i-1}^{\frac{1}{d p^{\prime}}} a_{i-1} \\
b_{i} \leq \frac{c_{2}\left(2^{\frac{d}{d-1}}\right)^{i}}{\eta^{\frac{d}{d-1}}} a_{i-1}^{\frac{d}{d-1}},
\end{array}\right.
$$

where $p^{\prime}:=p /(p-1)$.

Let us start with the second inequality. For every $\eta / 2<\delta<\varepsilon<\eta$, the isoperimetric inequality (3.18) together with inequality (3.14) entails

$$
|\Omega(\delta, \varepsilon)|^{\frac{d-1}{d}} \leq C_{d, \beta}\left(1+2^{p}\right)[h(\varepsilon)+h(\delta)],
$$

where $C_{d, \beta}:=C_{d} \beta_{2} / \beta_{1}$. Integrating in $\varepsilon$ between $\left[\varepsilon_{i}, \varepsilon_{i-1}\right]$ we get

$$
\int_{\varepsilon_{i}}^{\varepsilon_{i-1}}|\Omega(\delta, \varepsilon)|^{\frac{d-1}{d}} d \varepsilon \leq C_{d, \beta}\left(1+2^{p}\right)\left[\int_{\varepsilon_{i}}^{\varepsilon_{i-1}} h(\varepsilon) d \varepsilon+h(\delta)\left(\varepsilon_{i-1}-\varepsilon_{i}\right)\right],
$$

so that, recalling the definition of $\varepsilon_{i}$ in (3.15) 


$$
\left|\Omega\left(\delta, \varepsilon_{i}\right)\right|^{\frac{d-1}{d}}\left[\frac{1}{6} 2^{-i+1} \eta-\frac{1}{6} 2^{-i} \eta\right] \leq C_{d, \beta}\left(1+2^{p}\right)\left[\int_{\varepsilon_{i}}^{\varepsilon_{i}-1} h(\varepsilon) d \varepsilon+h(\delta)\left(\frac{1}{6} 2^{-i+1} \eta-\frac{1}{6} 2^{-i} \eta\right)\right] .
$$

Integrating in $\delta$ on $\left[\delta_{i-1}, \delta_{i}\right]$ we deduce

$$
\begin{aligned}
& \left|\Omega\left(\delta_{i}, \varepsilon_{i}\right)\right|^{\frac{d-1}{d}}\left[\frac{1}{6} 2^{-i+1} \eta-\frac{1}{6} 2^{-i} \eta\right]^{2} \\
& \quad \leq C_{d, \beta}\left(1+2^{p}\right)\left[\frac{1}{6} 2^{-i+1} \eta-\frac{1}{6} 2^{-i} \eta\right]\left[\int_{\varepsilon_{i}}^{\varepsilon_{i-1}} h(\varepsilon) d \varepsilon+\int_{\delta_{i-1}}^{\delta_{i}} h(\delta) d \delta\right] .
\end{aligned}
$$

We get

$$
\left|\Omega\left(\delta_{i}, \varepsilon_{i}\right)\right|^{\frac{d-1}{d}} \leq C_{d, \beta}\left(1+2^{p}\right) \frac{6 \cdot 2^{i}}{\eta}\left[\int_{\varepsilon_{i}}^{\varepsilon_{i-1}} h(\varepsilon) d \varepsilon+\int_{\delta_{i-1}}^{\delta_{i}} h(\delta) d \delta\right],
$$

so that the second inequality in (3.19) follows.

Let us come to the first inequality. Notice that for every $\eta / 2<\delta<\varepsilon<\eta$, the coarea formula, inequality (3.20), and the main inequality (3.14) entail

$$
\begin{aligned}
\int_{\delta}^{\varepsilon} h(s) d s & =\int_{\Omega(\delta, \varepsilon)}|\nabla u| d x \leq|\Omega(\delta, \varepsilon)|^{\frac{1}{p^{\prime}}}\left(\int_{\Omega(\delta, \varepsilon)}|\nabla u|^{p} d x\right)^{\frac{1}{p}} \\
& \leq|\Omega(\delta, \varepsilon)|^{\frac{1}{p^{\prime}}}\left(L \beta_{2}\right)^{\frac{1}{p}} \varepsilon h(\varepsilon)^{\frac{1}{p}}=|\Omega(\delta, \varepsilon)|^{\frac{1}{p^{\prime} d}}|\Omega(\delta, \varepsilon)|^{\frac{d-1}{p^{\prime} d}}\left(L \beta_{2}\right)^{\frac{1}{p}} \varepsilon h(\varepsilon)^{\frac{1}{p}} \\
& \leq|\Omega(\delta, \varepsilon)|^{\frac{1}{p^{\prime} d}}\left[C_{d, \beta}\left(1+2^{p}\right)\right]^{\frac{1}{p^{\prime}}}[h(\varepsilon)+h(\delta)]^{\frac{1}{p^{\prime}}}\left(L \beta_{2}\right)^{\frac{1}{p}} \varepsilon h(\varepsilon)^{\frac{1}{p}} \\
& \leq|\Omega(\delta, \varepsilon)|^{\frac{1}{p^{\prime} d}}\left[C_{d, \beta}\left(1+2^{p}\right)\left(L \beta_{2}\right)^{\frac{p^{\prime}}{p}}\right]^{\frac{1}{p^{\prime}}} \varepsilon[h(\varepsilon)+h(\delta)] .
\end{aligned}
$$

Integrating in $\varepsilon$ on $\left[\varepsilon_{i}, \varepsilon_{i-1}\right]$ we get

$$
\int_{\varepsilon_{i}}^{\varepsilon_{i-1}}\left[\int_{\delta}^{\varepsilon} h(s) d s\right] d \varepsilon \leq\left[C_{d, \beta}\left(1+2^{p}\right)\left(L \beta_{2}\right)^{\frac{p^{\prime}}{p}}\right]^{\frac{1}{p^{\prime}}}\left|\Omega\left(\delta, \varepsilon_{i-1}\right)\right|^{\frac{1}{p^{\prime} d}} \varepsilon_{i-1}\left[\int_{\varepsilon_{i}}^{\varepsilon_{i-1}} h(\varepsilon) d \varepsilon+h(\delta)\left[\varepsilon_{i-1}-\varepsilon_{i}\right]\right]
$$

so that

$$
\int_{\delta}^{\varepsilon_{i}} h(s) d s\left[\frac{1}{6} 2^{-i} \eta\right] \leq\left[C_{d, \beta}\left(1+2^{p}\right)\left(L \beta_{2}\right)^{\frac{p^{\prime}}{p}}\right]^{\frac{1}{p^{\prime}}}\left|\Omega\left(\delta, \varepsilon_{i-1}\right)\right|^{\frac{1}{p^{\prime} d}} \varepsilon_{i-1}\left[\int_{\varepsilon_{i}}^{\varepsilon_{i-1}} h(\varepsilon) d \varepsilon+h(\delta) \frac{1}{6} 2^{-i} \eta\right] .
$$

Integrating now in $\delta$ on $\left[\delta_{i-1}, \delta_{i}\right]$ we obtain

$$
\int_{\delta_{i}}^{\varepsilon_{i}} h(s) d s\left[\frac{1}{6} 2^{-i} \eta\right]^{2} \leq\left[C_{d, \beta}\left(1+2^{p}\right)\left(L \beta_{2}\right)^{\frac{p^{\prime}}{p}}\right]^{\frac{1}{p^{\prime}}}\left|\Omega\left(\delta_{i-1}, \varepsilon_{i-1}\right)\right|^{\frac{1}{p^{\prime} d}} \varepsilon_{i-1}\left[\frac{1}{6} 2^{-i} \eta\right]\left[\int_{\varepsilon_{i}}^{\varepsilon_{i-1}} h(\varepsilon) d \varepsilon+\int_{\delta_{i-1}}^{\delta_{i}} h(\delta) d \delta\right]
$$

which imply the first inequality in (3.19) since $\varepsilon_{i-1} \leq \eta$.

Step 2: Combining the main inequalities. We claim that we can find $\alpha>0$ such that setting

$$
U_{i}:=a_{i}^{\alpha} b_{i}
$$


we have for $i \geq 1$

$$
U_{i} \leq \frac{\tilde{c}}{\eta^{\frac{d}{d-1}}} A^{i} U_{i-1}^{\vartheta}
$$

where $\tilde{c}, A>0$, and $\vartheta>1$.

Indeed using (3.19) we have for $\alpha>0$ and $i \geq 1$

$$
a_{i}^{\alpha} b_{i} \leq \frac{c_{1}^{\alpha} c_{2}}{\eta^{\frac{d}{d-1}}}\left[2^{\alpha+\frac{d}{d-1}}\right]^{i} b_{i-1}^{\frac{\alpha}{d p^{\prime}}} a_{i-1}^{\alpha+\frac{d}{d-1}} .
$$

Writing

$$
\left\{\begin{array}{l}
\alpha+\frac{d}{d-1}=\vartheta \alpha \\
\frac{\alpha}{p^{\prime} d}=\vartheta
\end{array}\right.
$$

we obtain

$$
\alpha=\frac{1+\sqrt{1+\frac{4}{p^{\prime}(d-1)}}}{\frac{2}{p^{\prime} d}}
$$

and

$$
\vartheta=\frac{1+\sqrt{1+\frac{4}{p^{\prime}(d-1)}}}{2}>1,
$$

so that inequality (3.21) follows

Step 3: Decay for $E(\varepsilon)$. We claim that there exists $\varepsilon_{0}>0$ and $c_{0}>0$ such that for $\varepsilon \leq \varepsilon_{0}$

$$
E(\varepsilon) \leq c_{0} \varepsilon^{p} .
$$

Indeed from the inequality $E(\varepsilon) \leq L \beta_{2} \varepsilon^{p} h(\varepsilon)$ we infer using the coarea formula

$$
\begin{aligned}
\varepsilon E(\varepsilon) & \leq \int_{\varepsilon}^{2 \varepsilon} E(s) d s \leq \int_{\varepsilon}^{2 \varepsilon} L \beta_{2} s^{p} h(s) d s \leq L \beta_{2} 2^{p} \varepsilon^{p} \int_{\varepsilon}^{2 \varepsilon} h(s) d s \\
& =L \beta_{2} 2^{p} \varepsilon^{p} \int_{\Omega(\varepsilon, 2 \varepsilon)}|\nabla u| d x \leq L \beta_{2} 2^{p} \varepsilon^{p}\left(\int_{\Omega(\varepsilon, 2 \varepsilon)}|\nabla u|^{p} d x\right)^{\frac{1}{p}}|\Omega(\varepsilon, 2 \varepsilon)|^{\frac{1}{p^{\prime}}}
\end{aligned}
$$

so that

$$
E(\varepsilon) \leq L \beta_{2} 2^{p} \varepsilon^{p-1}|\Omega(\varepsilon, 2 \varepsilon)|^{\frac{1}{p^{\prime}}} E(2 \varepsilon)^{\frac{1}{p}} .
$$

Let $\varepsilon_{0}>0$ be such that

$$
L \beta_{2} 2^{p}\left|\Omega\left(0,2 \varepsilon_{0}\right)\right|^{\frac{1}{p^{\prime}}} \leq 1 .
$$

Then for $\varepsilon \leq \varepsilon_{0}$ we have

$$
E(\varepsilon) \leq \varepsilon^{p-1} E(2 \varepsilon)^{\frac{1}{p}}
$$

and (3.23) is a consequence of Lemma 3.6 below.

Step 4: Conclusion. Using the notation of Step 3, we claim that we can find $\eta$ so small that

$$
U_{0} \leq\left(\frac{\tilde{c}}{\eta^{\frac{d}{d-1}}}\right)^{-\frac{1}{\vartheta-1}} A^{-\frac{1}{(\vartheta-1)^{2}}}=\tilde{c}^{-\frac{1}{\vartheta-1}} A^{-\frac{1}{(\vartheta-1)^{2}}} \eta^{\alpha},
$$


where $\alpha$ is given in (3.22). Then it is easily seen by induction that $U_{i} \leq A^{-\frac{i}{\vartheta-1}} U_{0}$ so that

$$
\lim _{i \rightarrow+\infty} U_{i}=0
$$

and equality (3.17), concluding the proof of the theorem.

In order to verify that (3.24) can be achieved for $\eta$ small enough, we write

$$
\begin{aligned}
U_{0} & =\left|\Omega\left(\frac{\eta}{2}, \eta\right)\right|\left[\int_{\eta / 2}^{\eta} h(s) d s\right]^{\alpha}=\left|\Omega\left(\frac{\eta}{2}, \eta\right)\right|\left[\int_{\Omega\left(\frac{\eta}{2}, \eta\right)}|\nabla u| d x\right]^{\alpha} \\
& \leq\left|\Omega\left(\frac{\eta}{2}, \eta\right)\right|\left[E(\eta)^{\frac{1}{p}}\left|\Omega\left(\frac{\eta}{2}, \eta\right)\right|^{\frac{1}{p^{\prime}}}\right]^{\alpha}=\left|\Omega\left(\frac{\eta}{2}, \eta\right)\right|^{1+\frac{\alpha}{p^{\prime}}} E(\eta)^{\frac{\alpha}{p}}
\end{aligned}
$$

so that thanks to the decay for $E(\eta)$ obtained in Step 3 we deduce

$$
U_{0} \leq \hat{C}_{0}\left|\Omega\left(\frac{\eta}{2}, \eta\right)\right|^{1+\frac{\alpha}{p^{\prime}}} \eta^{\alpha}
$$

for some $\hat{C}_{0}>0$. Then (3.24) is achieved if $\eta$ is so small that

$$
\hat{C}_{0}\left|\Omega\left(\frac{\eta}{2}, \eta\right)\right|^{1+\frac{\alpha}{p^{\prime}}} \leq \tilde{c}^{-\frac{1}{\vartheta-1}} A^{-\frac{1}{(\vartheta-1)^{2}}}
$$

In the previous proof, we made use of the following simple lemma.

Lemma 3.6. Let $\varphi:\left[0,+\infty\left[\rightarrow\left[0,+\infty\left[\right.\right.\right.\right.$ be a monotone function such that for every $r \leq r_{0}$

$$
\varphi(r) \leq(2 r)^{\alpha} \varphi(2 r)^{\beta},
$$

where $r_{0}, \alpha>0$ and $0<\beta<1$. Then there exists $C>0$ such that for $r \leq r_{0}$

$$
\varphi(r) \leq C r^{\frac{\alpha}{1-\beta}} .
$$

Proof. Let $r \leq r_{0}$ and $m \in \mathbb{N}$ such that $\frac{r_{0}}{2}<2^{m} r \leq r_{0}$. We can write

$$
\begin{aligned}
\varphi(r) & \leq(2 r)^{\alpha} \varphi(2 r)^{\beta} \leq(2 r)^{\alpha}\left[(4 r)^{\alpha} \varphi(4 r)^{\beta}\right]^{\beta} \\
& \leq \cdots \leq\left[2^{1+2 \beta+3 \beta^{2}+\cdots+m \beta^{m-1}} r^{1+\beta+\beta^{2}+\cdots+\beta^{m-1}}\right]^{\alpha} \varphi\left(2^{m} r\right)^{\beta^{m}} \\
& \leq \tilde{C} r^{\frac{\alpha}{1-\beta}} r^{-\frac{\beta^{m} \alpha}{1-\beta}} \leq \hat{C} r^{\frac{\alpha}{1-\beta}}\left(2^{-m-1} r_{0}\right)^{-\frac{\beta^{m} \alpha}{1-\beta}} \leq C r^{\frac{\alpha}{1-\beta}},
\end{aligned}
$$

and the result follows.

Remark 3.7. An inspection of the previous proof shows that the estimate from below on the support is a consequence of the following inequality (according to the notation introduced above)

$$
E(\varepsilon)+c_{1} \delta^{p} \gamma(\delta, \varepsilon) \leq c_{2} \varepsilon^{p} h(\varepsilon) \quad \text { for a.e. } 0<\delta<\varepsilon \leq \varepsilon_{0},
$$

where $c_{1}, c_{2}>0$, which is a consequence of the comparison between $u$ and $u 1_{\{u \geq \varepsilon\}}$ ( $\varepsilon_{0}$ smaller than the constants appearing in (3.2)).

Remark 3.8 (Bound from below and the Hopf Lemma). In the classical setting with $p=2, f(x, \xi)=|\xi|^{2}$ and $\beta(x)=\beta$, the bound from below of $u$ on the associated regular domain $\Omega$ is a consequence of the classical Hopf lemma in view of the Robin condition at the boundary. Let indeed the subsolution $u \in W^{1,2}(\Omega), u \geq 0$, be associated to $\Omega$. We get easily that $u$ is subharmonic in $\Omega$. Let $x_{0}$ be a minimum point of $u$ on $\bar{\Omega} \backslash B$. We can assume $x_{0} \in \partial \Omega$, otherwise the bound from below is trivial. Since by Hopf Lemma $\frac{\partial u}{\partial v}\left(x_{0}\right)<0$, the Robin condition entails

$$
\beta u\left(x_{0}\right)=-\frac{\partial u}{\partial v}\left(x_{0}\right)>0
$$

so that $u \geq u\left(x_{0}\right)>0$ on $\Omega$. 
Remark 3.9. In the case $p=2$ and $\gamma>0$, the result of Theorem 3.5 was proved in [6] for subsolutions of

$$
u \mapsto \int_{\mathbb{R}^{d}}|\nabla u|^{2} d x+\beta \int_{J_{u}}\left[\left(u^{+}\right)^{2}+\left(u^{-}\right)^{2}\right] d \mathcal{H}^{d-1}+\gamma|\{u>0\}|,
$$

where $\beta>0$. In this paper, based on an iteration technique à la De Giorgi, we also cover the limit case $\gamma=0$.

We can now show that the free discontinuity problem is well posed.

Theorem 3.10. Let $u$ be the function given by Proposition 3.3. Then $u \in \mathcal{F}_{B, g}(D)$, and it is a minimizer for the free discontinuity functional $F$ in (3.6).

Proof. The $S B V$ regularity of $u$ follows by the chain rule formula in $B V$ in view of the lower bound (3.12) and of the fact $u^{p} \in S B V\left(\mathbb{R}^{d}\right)$. The minimality is a consequence of Proposition 3.3.

The following result is fundamental to prove some regularity for minimizers of $F$.

Proposition 3.11 (Almost-quasi minimality). Let $u$ be a minimizer of $F$ on $\mathcal{F}_{B, g}(D)$ with $0 \leq u \leq\|g\|_{\infty}$. Then there exists $\alpha>0$ with

$$
u>\alpha \quad \text { a.e. on } \operatorname{supp}(u)
$$

and such that function $\left(2 \beta_{1}\right)^{1 / p} \alpha u$ is an almost-quasi minimizer of the Mumford-Shah functional

$$
M S(u):=\int_{D} f(x, \nabla u) d x+\mathcal{H}^{d-1}\left(J_{u}\right)
$$

on D with Dirichlet condition $u=g$ on B according to Definition 2.2.

Proof. The existence of $\alpha>0$ satisfying (3.26) is a consequence Theorem 3.5. Coming to almost-quasi minimality, let $B_{\rho}(x) \subset D$ and $v \in S B V_{l o c}(D)$ be such that $v=g$ on $B$ and $\{v \neq u\} \subseteq B_{\rho}(x) \subseteq D$. Let us consider

$$
w:=\left(v \wedge\|g\|_{\infty}\right) \vee 0 .
$$

Clearly $w \in S B V(D)$ with $\{w \neq u\} \subseteq B_{\rho}(x)$ and still $w=g$ on $B$. Comparing $u$ and $w$ we get

$$
\begin{aligned}
& \int_{B_{\rho}(x)} f(x, \nabla u) d x+2 \beta_{1} \alpha^{p} \mathcal{H}^{d-1}\left(J_{u} \cap \bar{B}_{\rho}(x)\right) \\
& \quad \leq \int_{B_{\rho}(x)} f(x, \nabla w) d x+2 \beta_{2}\|g\|_{\infty}^{p} \mathcal{H}^{d-1}\left(J_{w} \cap \bar{B}_{\rho}(x)\right)+\gamma \omega_{d} r^{d}
\end{aligned}
$$

so that

$$
\begin{aligned}
& \int_{B_{\rho}(x)} f(x, \nabla u) d x+2 \beta_{1} \alpha^{p} \mathcal{H}^{d-1}\left(J_{u} \cap \bar{B}_{\rho}(x)\right) \\
& \quad \leq \int_{B_{\rho}(x)} f(x, \nabla v) d x+2 \beta_{2}\|g\|_{\infty}^{p} \mathcal{H}^{d-1}\left(J_{v} \cap \bar{B}_{\rho}(x)\right)+\gamma \omega_{d} r^{d},
\end{aligned}
$$

and the result follows.

We complete our analysis of the free discontinuity functional $F$ with the following density result.

Proposition 3.12. Given $v \in \mathcal{F}_{B, g}(D) \cap L^{\infty}\left(\mathbb{R}^{d}\right)$ with $\mathcal{H}^{d-1}\left(J_{v}\right)<+\infty$, for every $\varepsilon>0$ there exists $w \in \mathcal{F}_{B, g}(D) \cap$ $L^{\infty}\left(\mathbb{R}^{d}\right)$ with $J_{w} \subset \subset D \backslash \bar{B}, \mathcal{H}^{d-1}\left(J_{w}\right)<+\infty$, and such that

$$
F(w) \leq F(v)+\varepsilon .
$$


Proof. We can assume that $g \in C_{c}^{1}(D)$ with $g \geq 0$. Let $\varepsilon_{1}>0$ to be fixed below, and let $U \subset \subset D \backslash \bar{B}$ open such that

$$
\mathcal{H}^{d-1}\left(J_{v} \cap(D \backslash U)\right)<\varepsilon_{1} .
$$

Since $D \backslash \bar{B}$ has Lipschitz boundary, for every $x \in \partial D \cup \partial B$ we can find an orthogonal coordinate system $y=\left(y^{\prime}, y_{d}\right)$ with origin at $x$, two numbers $r, s>0$, and a Lipschitz function $f^{x}: \mathbb{R}^{d-1} \rightarrow \mathbb{R}$ such that setting

$$
V_{r, s}^{x}:=\left\{\left|y^{\prime}\right|<r,\left|y_{d}\right|<s\right\}
$$

we have $V_{r, s}^{x} \cap U=\emptyset$ and

$$
(D \backslash \bar{B}) \cap V_{r, s}^{x}=\left\{y \in V_{r, s}^{x}: y_{d}<f^{x}\left(y^{\prime}\right)\right\} .
$$

We can also assume that for $\xi>0$ small enough

$$
\left\{\left|y^{\prime}\right|<r, f^{x}\left(y^{\prime}\right)-\xi<y_{d}<f^{x}\left(y^{\prime}\right)+\xi\right\} \subseteq V_{r, s}^{x} .
$$

By compactness, we can cover $\partial D \cup \partial B$ with a finite number of neighborhoods $V_{1}, \ldots, V_{m}$ of the type $V_{i}:=V_{r_{i}}^{x_{i}} s_{i}$, and associated function $f_{i}$. Moreover it is not restrictive to assume that the $\mathcal{H}^{d-1}$-measure of the part of $\partial D$ on which the $V_{i}$ 's overlap is less than $\varepsilon_{1}$. Let $\psi_{1}, \ldots, \psi_{m}$ be a partition of unity for $\partial D$ subordinated to the $V_{i}$ 's. We write

$$
v=\psi_{1} v+\cdots+\psi_{m} v+\left(1-\psi_{1}-\cdots-\psi_{m}\right) v:=v_{1}+\cdots+v_{m}+v_{0}
$$

Notice that $v_{0}=v$ on $U$, and that $v_{i}$ has compact support in $V_{i}$ (so that we can assume that they are defined on the entire $\mathbb{R}^{d}$ for the operations performed below).

We proceed to approximate each $v_{i}$ by "pushing inside" the jumps occurring at the boundary. Given $\xi>0$ small, we consider

$$
v_{i}^{\xi}(y):= \begin{cases}v_{i}\left(y^{\prime}, y_{d}+\xi\right) & \text { if } y_{d}<f_{i}\left(y^{\prime}\right)-\xi \\ \left(\psi_{i} g\right)\left(y^{\prime}, f_{i}\left(y^{\prime}\right)\right) & \text { if } y_{d} \geq f_{i}\left(y^{\prime}\right)-\xi\end{cases}
$$

Let us consider

$$
v^{\xi}:=v_{1}^{\xi}+\cdots+v_{m}^{\xi}+v_{0}
$$

By construction, $v^{\xi} \in S B V\left(\mathbb{R}^{d}\right) \cap L^{\infty}\left(\mathbb{R}^{d}\right)$ with the jump set with finite $\mathcal{H}^{d-1}$-measure, and well contained in $D$, being $v^{\xi}$ Lipschitz regular in a neighborhood of $\partial D$.

Concerning the behavior of the functional $F$, for $\xi \rightarrow 0$ we get that the volume energy of $v^{\xi}$ and the measure of its support are arbitrarily closed to that of $v$. As for the surface energy, which we denote by $\mathcal{E}^{s}$, thanks to the continuity of $\beta(x)$ we get with obvious notation

$$
\mathcal{E}^{s}\left(v^{\xi}\right) \leq \mathcal{E}^{s}\left(v_{0}, U\right)+\mathcal{E}^{s}(v, \partial D \cup \partial B)+C \varepsilon_{1},
$$

where $C$ depends only on $m, g$ and $\|v\|_{\infty}$. The last term takes into account the jumps in $(D \backslash \bar{B}) \backslash U$, and the possible interference of the $v_{i}^{\xi}$ obtained by the translation of the $v_{i}$ 's. The result thus follows if $C \varepsilon_{1}<\varepsilon$ and $\xi$ is small enough.

We are now in a position to prove the main result of the paper.

Proof of Theorem 3.2. Let $u$ be a minimizer of $F$ on $\mathcal{F}_{B, g}(D)$ with $0 \leq u \leq\|g\|_{\infty}$ : its existence is secured by Proposition 3.10. Thanks to Proposition 3.11 and to Theorem 2.3, we deduce that

$$
\mathcal{H}^{d-1}\left(\left(\overline{J_{u}} \backslash J_{u}\right) \cap D\right)=0,
$$

i.e., the jump set of $u$ is essentially closed in $D$. Moreover the lower bound (3.26) entails

$$
\mathcal{H}^{d-1}\left(J_{u}\right)<+\infty \text {. }
$$

Let $\Omega$ be given by the connected component of $D \backslash \overline{J_{u}}$ on which $u$ does not vanish (we have just one component by minimality). Recalling (3.28), and since

$$
\partial \Omega \subseteq\left(\overline{J_{u}} \cap D\right) \cup \partial D
$$


we get that $\Omega \in \mathcal{A}_{B}(D)$. Moreover

$$
\int_{\partial \Omega \backslash J_{u}}\left[\left(u^{+}\right)^{p}+\left(u^{-}\right)^{p}\right] d \mathcal{H}^{d-1}=0 .
$$

Indeed thanks to (3.27)

$$
\mathcal{H}^{d-1}\left(\left(\partial \Omega \backslash J_{u}\right) \cap D\right)=0,
$$

while

$$
u^{ \pm}=0 \quad \mathcal{H}^{d-1} \text {-a.e. on }\left(\partial \Omega \backslash J_{u}\right) \cap \partial D
$$

since, being $D$ Lipschitz, we have $u^{-}=0 \mathcal{H}^{d-1}$-a.e. on $\partial D$.

Since

$$
u_{\mid \Omega} \in W^{1, p}(\Omega),
$$

and taking into account (3.29), we conclude that

$$
J(\Omega) \leq \int_{\Omega} f(x, \nabla u) d x+\int_{\partial \Omega} \beta(x)\left[\left|u^{+}\right|^{p}+\left|u^{-}\right|^{p}\right] d \mathcal{H}^{d-1}=F(u) .
$$

Let us prove that $\Omega$ is a minimizer of the shape optimization problem (3.4). Let us consider $\tilde{\Omega} \in \mathcal{A}_{B}(D)$ with associated function $v \in W^{1, p}(\tilde{\Omega}) \cap L^{\infty}(\tilde{\Omega})$ which realizes $J(\tilde{\Omega})$. By extending $v$ to zero outside $\tilde{\Omega}$ we get $v \in$ $\mathcal{F}_{B, g}(D), J_{v} \subseteq \partial \tilde{\Omega}$, and

$$
\begin{aligned}
F(v) & =\int_{\mathbb{R}^{d}} f(x, \nabla v) d x+\int_{J_{v}} \beta(x)\left[\left|v^{+}\right|^{p}+\left|v^{-}\right|^{p}\right] d \mathcal{H}^{d-1} \\
& \leq \int_{\tilde{\Omega}} f(x, \nabla v) d x+\int_{\partial \tilde{\Omega}} \beta(x)\left[\left|v^{+}\right|^{p}+\left|v^{-}\right|^{p}\right] d \mathcal{H}^{d-1}=J(\tilde{\Omega})
\end{aligned}
$$

so that in view of (3.30) we infer $J(\Omega) \leq J(\tilde{\Omega})$ and the optimality of $\Omega$ follows. Moreover we infer $J(\Omega)=F(u)$.

Let us finally prove the "relaxation condition" (3.5). By Proposition 3.12, for every $\varepsilon>0$ we can find $w \in$ $\mathcal{F}_{B, g}(D) \cap L^{\infty}\left(\mathbb{R}^{d}\right)$ with $J_{w}$ well contained in $D \backslash \bar{B}$ and with finite $\mathcal{H}^{d-1}$-measure, and such that

$$
F(w)<F(u)+\varepsilon .
$$

By the density result Theorem 2.4, truncating and using a cut-off function to accommodate the Dirichlet condition on $B$, we can find $w_{k} \in \mathcal{F}_{B, g}(D) \cap L^{\infty}\left(\mathbb{R}^{d}\right)$ with $J_{w_{k}} \subset \subset D \backslash \bar{B}$ essentially closed and polyhedral (i.e., given by the union of the intersection with $D$ of a finite number of $(d-1)$-simplexes), $w_{k}$ is of class $W^{1, p}$ on $D \backslash \bar{J}_{w_{k}}$ for every $k$, and such that

$$
\begin{aligned}
& w_{k} \rightarrow w \quad \text { strongly in } L^{p}(D), \\
& \nabla w_{k} \rightarrow \nabla w \quad \text { strongly in } L^{p}\left(D ; \mathbb{R}^{d}\right),
\end{aligned}
$$

and

$$
F\left(w_{k}\right) \rightarrow F(w) .
$$

Note that the convergence for the measure of the supports can be obtained by replacing $w_{k}$ given by Theorem 2.4, if necessary, by $\left(w_{k}-\eta_{k}\right)_{+}$for suitable $\eta_{k} \rightarrow 0$.

If we enlarge the jump set creating small holes $H_{k}$ with polyhedral boundary, we get that the domain

$$
\Omega_{k}:=D \backslash \overline{H_{k}} \in \mathcal{A}_{B}(D)
$$


is Lipschitz regular. The restriction of $w_{k}$ to $\Omega_{k}$ is moreover an admissible function to compute $J\left(\Omega_{k}\right)$. We can thus consider the holes so small and $k$ so large that

$$
J\left(\Omega_{k}\right) \leq \int_{\Omega_{k}} f\left(x, \nabla w_{k}\right) d x+\int_{\partial \Omega_{k}} \beta(x)\left|w_{k}\right|^{p} d \mathcal{H}^{d-1} \leq F\left(w_{k}\right)+\varepsilon \leq J(u)+2 \varepsilon .
$$

Now the result follows by letting $\varepsilon=\varepsilon_{n} \rightarrow 0$ and selecting the associated $k=k_{n}$.

\section{Essential closedness of the jump set of almost-quasi minimizers of free discontinuity functionals}

The present section is devoted to the proof of Theorem 2.3. As explained in Section 2, we follow the approach a la De Giorgi-Carriero-Leaci [10] along the lines of [2, Chapter 7]. The main point is to recover a decay lemma for the energy (see Theorem 4.8). This is achieved through a contradiction argument in which the analysis of sequences of almost-quasi minimizers with vanishing jump set play a key role. As in the classical setting, we need to prove that our weaker minimality still entails that they converge to a function without jump which is a local minimizer of the volume energy.

We divide the section in several parts. In Subsection 4.1 we collect some regularity results for local minimizers of integral functionals. In Subsection 4.2 we prove some technical lemmas concerning the behavior of almost-quasi minimizers on the unit ball with vanishing jump set. In Subsection 4.3 we prove the basic decay lemma, while Subsection 4.4 contains the proof of Theorem 2.3.

\subsection{Regularity results for local minimizers of integral functionals}

We will need suitable gradient bound estimates for local minimizers of the bulk energy. Let $f: \mathbb{R}^{d} \rightarrow[0,+\infty]$. For every $r \leq 1$ and $u \in W^{1, p}\left(B_{r}\right)$, let

$$
F\left(u, B_{r}\right):=\int_{B_{r}} f(\nabla u) d x
$$

Definition 4.1 (Local minimizers). Let $r>0$. We say that $u \in W^{1, p}\left(B_{r}\right)$ is a local minimizer of $F\left(\cdot, B_{r}\right)$ if

$$
F\left(u, B_{r}\right) \leq F\left(v, B_{r}\right)
$$

for every $v \in W^{1, p}\left(B_{r}\right)$ with $\{u \neq v\} \subset \subset B_{r}$.

The following result has been proved in [11, Theorem 2.2].

Theorem 4.2 (Interior gradient bound). Assume that $f: \mathbb{R}^{d} \rightarrow \mathbb{R}$ is a continuous function satisfying $\left(H_{1}\right)$ and $\left(H_{2}\right)$. Let $u \in W^{1, p}\left(B_{1}\right)$ be a local minimizer of $F\left(\cdot, B_{1}\right)$. Then $u$ is locally Lipschitz in $B_{1}$, and there exists a constant $C_{0}=C_{0}(d, p, L, \mu)>0$ such that

$$
\sup _{B_{\rho / 2}}|\nabla u|^{p} \leq \frac{C_{0}}{\rho^{d}} \int_{B_{\rho}}|\nabla u|^{p} d x \quad \text { for every } \rho \leq 1 .
$$

We need an analogous result for the case with boundary conditions. Let us introduce, for $\delta \geq 0$,

$$
H_{\delta}:=\left\{x=\left(x^{\prime}, x_{d}\right) \in \mathbb{R}^{d}: x_{d}>-\delta\right\},
$$

and for every $r \leq 1$ and $u \in W^{1, p}\left(B_{r}\right)$, let

$$
F_{0, \delta}\left(u, B_{r}\right):= \begin{cases}\int_{B_{r}} f(\nabla u) d x & \text { if } u=0 \text { a.e. in } B_{r} \backslash H_{\delta}, \\ +\infty & \text { otherwise. }\end{cases}
$$

The definition of local minimizers is adapted to $F_{0, \delta}$, taking into account the boundary condition. The following result has been proved in [3, Theorem 3.8]. 
Theorem 4.3 (Boundary gradient bound). Assume that $\delta \in[0,1 / 2]$ and that $f: \mathbb{R}^{d} \rightarrow \mathbb{R}$ is a continuous function satisfying $\left(H_{1}\right)$ and $\left(H_{2}\right)$. Let $u \in W^{1, p}\left(B_{1}\right)$ be a local minimizer of $F_{0, \delta}\left(\cdot, B_{1}\right)$. Then, for each $R_{0}<1$, there exists a constant $C_{0}^{\prime}=C_{0}^{\prime}\left(d, p, L, \mu, R_{0},\|\nabla u\|_{p}\right)>0$ (independent of $\delta$ ) such that $u$ is locally Lipschitz continuous in $B_{1}$ and

$$
\sup _{B_{\rho / 2}}|\nabla u|^{p} \leq C_{0}^{\prime}\left(\frac{1}{\rho^{d}} \int_{B_{\rho}}|\nabla u|^{p} d x+1\right) \quad \text { for every } \rho \leq R_{0} .
$$

\subsection{Some lemmas on the unit ball}

In the footsteps of [2, Chapter 7], we study the behavior of sequences of functions in $S B V\left(B_{1}\right)$ with vanishing jump set which satisfy a suitable minimality property for the functional (2.1).

More precisely, let $f_{n}: B_{1} \times \mathbb{R}^{d} \rightarrow[0,+\infty[$ be a Caratheodory function with

$$
\begin{aligned}
& f_{n}(x, \xi) \leq L\left(1+|\xi|^{p}\right) \\
& \xi \mapsto f_{n}(x, \xi) \text { is convex for a.e. } x \in B_{1},
\end{aligned}
$$

and such that

$$
f_{n} \rightarrow f_{\infty} \quad \text { uniformly on the compact sets of } B_{1} \times \mathbb{R}^{d} .
$$

Here $L>0$ and $p>1$. We are interested in the behavior of a sequence $\left(u_{n}\right)_{n \in \mathbb{N}}$ in $S B V\left(B_{1}\right)$ which satisfy the minimality property

$$
\begin{aligned}
& \int_{B_{r}} f_{n}\left(x, \nabla u_{n}\right) d x+c_{n} \mathcal{H}^{d-1}\left(J_{u_{n}} \cap \bar{B}_{r}\right) \\
& \quad \leq \int_{B_{r}} f_{n}(x, \nabla v) d x+\Lambda c_{n} \mathcal{H}^{d-1}\left(J_{v} \cap \bar{B}_{r}\right)+D_{n}(r)
\end{aligned}
$$

for every $v \in \operatorname{SBV}\left(B_{1}\right)$ with $\left\{v \neq u_{n}\right\} \subseteq B_{r}$ and $r \in[0,1]$. Here $\Lambda \geq 1, c_{n} \geq 0$ and $D_{n}:[0,1] \rightarrow \mathbb{R}^{+}$is such that

$$
D_{n} \rightarrow 0 \quad \text { pointwise. }
$$

Following [10], we will make use of a suitable truncation of a function in $\operatorname{SBV}\left(B_{1}\right)$. For every $s \in\left[0, \omega_{d}\right]$, let us set

$$
u_{*}\left(s, B_{1}\right):=\inf \{t \in \mathbb{R}:|\{u<t\}| \geq s\} .
$$

If

$$
\left(2 \gamma_{d} \mathcal{H}^{d-1}\left(J_{u}\right)\right)^{\frac{d}{d-1}}<\frac{\omega_{d}}{2},
$$

where $\gamma_{d}$ is the constant appearing in the relative isoperimetric inequality, let

$$
\tau^{-}\left(u, B_{1}\right):=u_{*}\left(\left(2 \gamma_{d} \mathcal{H}^{d-1}\left(J_{u}\right)\right)^{\frac{d}{d-1}}, B_{1}\right)
$$

and

$$
\tau^{+}\left(u, B_{1}\right):=u_{*}\left(\omega_{d}-\left(2 \gamma_{d} \mathcal{H}^{d-1}\left(J_{u}\right)\right)^{\frac{d}{d-1}}, B_{1}\right) .
$$

Notice that $\tau^{-}\left(u, B_{1}\right) \leq m \leq \tau^{+}\left(u, B_{1}\right)$ for every median $m$ of $u$, i.e.,

$$
\left|\left\{x \in B_{1}: u(x)<t\right\}\right| \leq \frac{\omega_{d}}{2} \quad \text { for every } t<m
$$

and

$$
\left|\left\{x \in B_{1}: u(x)>t\right\}\right| \leq \frac{\omega_{d}}{2} \quad \text { for every } t>m .
$$

The following result holds true (see [10, Theorem 3.5] and [2, Proposition 7.5]). 
Lemma 4.4. Let $\left(u_{n}\right)_{n \in \mathbb{N}}$ be a sequence in $\operatorname{SBV}\left(B_{1}\right)$ such that

$$
\sup _{n} \int_{B_{1}}|\nabla u|^{p} d x<+\infty \quad \text { and } \quad \lim _{n \rightarrow+\infty} \mathcal{H}^{d-1}\left(J_{u_{n}}\right)=0
$$

for some $p>1$, and let $m_{n}$ be medians of $u_{n}$. Then there exists a subsequence $\left(u_{n_{k}}\right)_{k \in \mathbb{N}}$ and $u \in W^{1, p}\left(B_{1}\right)$ such that

$$
u_{n_{k}}-m_{n_{k}} \rightarrow u \quad \text { a.e. in } B_{1} .
$$

Moreover the truncated function

$$
\bar{u}_{n_{k}}:=\left(u_{n_{k}} \vee \tau^{-}\left(u_{n_{k}}, B_{1}\right)\right) \wedge \tau^{+}\left(u_{n_{k}}, B_{1}\right)
$$

satisfies

$$
\bar{u}_{n_{k}}-m_{n_{k}} \rightarrow u \quad \text { strongly in } L^{p}\left(B_{1}\right)
$$

and

$$
\left|\left\{u_{n_{k}} \neq \bar{u}_{n_{k}}\right\}\right| \leq 2\left(2 \gamma_{d} \mathcal{H}^{d-1}\left(J_{u_{n_{k}}}\right)\right)^{\frac{d}{d-1}} .
$$

We begin with the following lemma.

Lemma 4.5. Let $f_{n}$ satisfy (4.1) and let $\left(u_{n}\right)_{n \in \mathbb{N}}$ be a sequence in $S B V\left(B_{1}\right)$ satisfying the minimality property (4.4) and such that

$$
\sup _{n}\left[\int_{B_{1}}\left|\nabla u_{n}\right|^{p} d x+c_{n} \mathcal{H}^{d-1}\left(J_{u_{n}}\right)\right]<+\infty \quad \text { and } \quad \mathcal{H}^{d-1}\left(J_{u_{n}}\right) \rightarrow 0 .
$$

Then there exists $E \subseteq[0,1]$ with $|[0,1] \backslash E|=0$ such that, up to a subsequence, the truncated functions $\bar{u}_{n}$ defined in (4.6) still satisfy the minimality property (4.4) for every $r \in E$, with $D_{n}$ replaced by $\tilde{D}_{n}: E \rightarrow \mathbb{R}^{+}$such that $\tilde{D}_{n} \rightarrow 0$ pointwise.

Proof. Thanks to Lemma 4.4, in view of assumption (4.8), we have

$$
c_{n}\left|\left\{u_{n} \neq \bar{u}_{n}\right\}\right| \leq \gamma_{d} c_{n}\left(\mathcal{H}^{d-1}\left(J_{u_{n}}\right)\right)^{\frac{d}{d-1}} \rightarrow 0 .
$$

Since

$$
\left|\left\{u_{n} \neq \bar{u}_{n}\right\}\right|=\int_{0}^{1} \mathcal{H}^{d-1}\left(\left\{u_{n} \neq \bar{u}_{n}\right\} \cap \partial B_{r}\right) d r
$$

we deduce that, up to a subsequence, for a.e. $r \in[0,1]$

$$
c_{n} \mathcal{H}^{d-1}\left(\left\{u_{n} \neq \bar{u}_{n}\right\} \cap \partial B_{r}\right) \rightarrow 0 .
$$

Let us consider now $v \in S B V\left(B_{1}\right)$ with $\left\{v \neq \bar{u}_{n}\right\} \subseteq B_{r}$, where $r$ satisfies the previous property. Comparing $u_{n}$ with $w:=v 1_{B_{r}}+u_{n} 1_{B_{1} \backslash B_{r}}$ we get

$$
\begin{aligned}
& \int_{B_{r}} f_{n}\left(x, \nabla u_{n}\right) d x+c_{n} \mathcal{H}^{d-1}\left(J_{u_{n}} \cap \bar{B}_{r}\right) \\
& \quad \leq \int_{B_{r}} f_{n}(x, \nabla v) d x+\Lambda c_{n} \mathcal{H}^{d-1}\left(J_{v} \cap B_{r}\right)+\Lambda c_{n} \mathcal{H}^{d-1}\left(\left\{v \neq u_{n}\right\} \cap \partial B_{r}\right)+D_{n}(r) \\
& \leq \int_{B_{r}} f_{n}(x, \nabla v) d x+\Lambda c_{n} \mathcal{H}^{d-1}\left(J_{v} \cap \bar{B}_{r}\right)+\Lambda c_{n} \mathcal{H}^{d-1}\left(\left\{u_{n} \neq \bar{u}_{n}\right\} \cap \partial B_{r}\right)+D_{n}(r) .
\end{aligned}
$$


Since in view of (4.1)

$$
\begin{aligned}
& \int_{B_{r}} f_{n}\left(x, \nabla \bar{u}_{n}\right) d x+c_{n} \mathcal{H}^{d-1}\left(J_{\bar{u}_{n}} \cap \bar{B}_{r}\right) \\
& \quad \leq \int_{B_{r}} f_{n}\left(x, \nabla u_{n}\right) d x+c_{n} \mathcal{H}^{d-1}\left(J_{u_{n}} \cap \bar{B}_{r}\right)+L\left|\left\{u_{n} \neq \bar{u}_{n}\right\}\right|,
\end{aligned}
$$

we conclude

$$
\int_{B_{r}} f_{n}\left(x, \nabla \bar{u}_{n}\right) d x+c_{n} \mathcal{H}^{d-1}\left(J_{\bar{u}_{n}} \cap \bar{B}_{r}\right) \leq \int_{B_{r}} f_{n}(x, \nabla v) d x+\Lambda c_{n} \mathcal{H}^{d-1}\left(J_{v} \cap \bar{B}_{r}\right)+\tilde{D}_{n}(r)
$$

where

$$
\tilde{D}_{n}(r):=L\left|\left\{u_{n} \neq \bar{u}_{n}\right\}\right|+\Lambda c_{n} \mathcal{H}^{d-1}\left(\left\{u_{n} \neq \bar{u}_{n}\right\} \cap \partial B_{r}\right)+D_{n}(r) \rightarrow 0,
$$

and the result follows.

Lemma 4.6. Let $f_{n}$ satisfy (4.1), (4.2) and (4.3). Let $\left(u_{n}\right)_{n \in \mathbb{N}}$ be a sequence in $S B V\left(B_{1}\right)$ satisfying the minimality property (4.4) such that

$$
\begin{gathered}
\sup _{n} \int_{B_{1}}\left|\nabla u_{n}\right|^{p} d x<+\infty, \quad \mathcal{H}^{d-1}\left(J_{u_{n}}\right) \rightarrow 0, \\
\sup _{n}\left[\int_{B_{1}} f_{n}\left(x, \nabla u_{n}\right) d x+c_{n} \mathcal{H}^{d-1}\left(J_{u_{n}}\right)\right]<+\infty
\end{gathered}
$$

and

$$
u_{n} \rightarrow u \in W^{1, p}\left(B_{1}\right) \quad \text { pointwise a.e. }
$$

Then $u$ is a local minimizer in $W^{1, p}\left(B_{1}\right)$ of the functional

$$
v \mapsto \int_{B_{1}} f_{\infty}(x, \nabla v) d x
$$

and for every $r \in[0,1]$

$$
\lim _{n \rightarrow+\infty} \int_{B_{r}} f_{n}\left(x, \nabla u_{n}\right) d x=\int_{B_{r}} f_{\infty}(x, \nabla u) d x \quad \text { and } \quad \lim _{n \rightarrow+\infty} c_{n} \mathcal{H}^{d-1}\left(J_{u_{n}} \cap \bar{B}_{r}\right)=0 .
$$

Proof. Let $\bar{u}_{n}$ denote the truncation of $u_{n}$ according to (4.6). We have thanks to (4.7), (4.9) and (4.10)

$$
c_{n}\left|\left\{\bar{u}_{n} \neq u_{n}\right\}\right|=c_{n} \int_{0}^{1} \mathcal{H}^{d-1}\left(\left\{\bar{u}_{n} \neq u_{n}\right\} \cap \partial B_{r}\right) d r \rightarrow 0 .
$$

By Helly's theorem, up to a subsequence we can assume that for every $r \in(0,1)$

$$
\lim _{n \rightarrow+\infty}\left[\int_{B_{r}} f_{n}\left(x, \nabla \bar{u}_{n}\right) d x+c_{n} \mathcal{H}^{d-1}\left(J_{\bar{u}_{n}} \cap \bar{B}_{r}\right)\right]=\alpha(r),
$$

where $\alpha:[0,1] \rightarrow[0,+\infty[$ is an increasing function.

In view of Lemma 4.4 we get easily

$$
\bar{u}_{n} \rightarrow u \quad \text { strongly in } L^{p}\left(B_{1}\right),
$$


and

$$
\left|\left\{\bar{u}_{n} \neq u_{n}\right\}\right| \rightarrow 0 .
$$

Following [12, Theorem 2.6] we have

$$
\int_{B_{r}} f_{\infty}(x, \nabla u) d x \leq \liminf _{n \rightarrow+\infty} \int_{B_{r}} f_{n}\left(x, \nabla \bar{u}_{n}\right) d x .
$$

Indeed, by Chacon biting lemma (see e.g. [2, Lemma 3.52]), there exists a decreasing family of Borel sets $A_{k} \subset B_{1}$ with $\left|A_{k}\right| \rightarrow 0$ as $k \rightarrow \infty$ and such that

$$
\left(\left|\nabla u_{n}\right|^{p} 1_{B_{1} \backslash A_{k}}\right)_{n \in \mathbb{N}} \text { is equintegrable. }
$$

Then for every $m \in \mathbb{N}$

$$
\begin{aligned}
\liminf _{n \rightarrow+\infty} \int_{B_{r}} f_{n}\left(x, \nabla \bar{u}_{n}\right) d x & \geq \liminf _{n \rightarrow+\infty} \int_{\left[B_{r} \backslash A_{k}\right] \cap M_{n, m}} f_{n}\left(x, \nabla \bar{u}_{n}\right) d x \\
& \geq \liminf _{n \rightarrow+\infty} \int_{B_{r} \backslash A_{k}} f_{\infty}\left(x, \nabla \bar{u}_{n}\right) d x-\limsup _{n \rightarrow+\infty} \int_{\left[B_{r} \backslash A_{k}\right] \backslash M_{n, m}} L\left(1+\left|\nabla \bar{u}_{n}\right|^{p}\right) d x-e(m)
\end{aligned}
$$

where

$$
M_{n, m}:=\left\{x \in B_{1}:\left|\nabla \bar{u}_{n}\right| \leq m\right\}
$$

and $e(m) \rightarrow 0$. Then, thanks to (4.13) and in view of the lower semicontinuity result [2, Theorem 5.29] we get

$$
\liminf _{n \rightarrow+\infty} \int_{B_{r} \backslash A_{k}} f_{\infty}\left(x, \nabla \bar{u}_{n}\right) d x \geq \int_{B_{r} \backslash A_{k}} f_{\infty}(x, \nabla u) d x,
$$

so that (4.15) follows by letting $m \rightarrow+\infty$ and then $k \rightarrow+\infty$.

In view of (4.15) and (4.14), for every $r \in(0,1)$ we obtain

$$
\int_{B_{r}} f_{\infty}(x, \nabla u) d x \leq \liminf _{n \rightarrow+\infty} \int_{B_{r}} f_{n}\left(x, \nabla \bar{u}_{n}\right) d x \leq \liminf _{n \rightarrow+\infty} \int_{B_{r}} f_{n}\left(x, \nabla u_{n}\right) d x
$$

Let us consider $v \in W^{1, p}\left(B_{1}\right)$ and $r \in(0,1)$ such that $\{v \neq u\} \subseteq B_{r}$. Considering the measures

$$
\mu_{n}:=c_{n} \mathcal{H}^{d-1}\left\lfloor J_{\bar{u}_{n}},\right.
$$

we can assume up to a subsequence that

$$
\mu_{n} \stackrel{*}{\rightarrow} \mu \quad \text { weakly* in the sense of measures }
$$

for some finite positive measure $\mu$ on $B_{1}$. Let us consider

$$
r<r^{\prime}<r^{\prime \prime}<1
$$

with

$$
\mu\left(\partial B_{r^{\prime}}\right)=0 \quad \text { and } \quad r^{\prime \prime} \in E,
$$

where $E \subseteq[0,1]$ is given by Lemma 4.5. Let $\varphi \in C_{c}^{\infty}\left(B_{1}\right)$ be a cut-off function between $B_{r^{\prime}}$ and $B_{r^{\prime \prime}}$, and let us compare $\bar{u}_{n}$ with $\varphi v+(1-\varphi) \bar{u}_{n}$. Since $r^{\prime \prime} \in E$ we can write

$$
\begin{aligned}
& \int_{B_{r^{\prime \prime}}} f_{n}\left(x, \nabla \bar{u}_{n}\right) d x+c_{n} \mathcal{H}^{d-1}\left(J_{\bar{u}_{n}} \cap \bar{B}_{r^{\prime \prime}}\right) \\
& \quad \leq \int_{B_{r^{\prime \prime}}} f_{n}\left(x, \varphi \nabla v+(1-\varphi) \nabla \bar{u}_{n}+\nabla \varphi\left(v-\bar{u}_{n}\right)\right) d x
\end{aligned}
$$




$$
\begin{aligned}
& +\Lambda c_{n} \mathcal{H}^{d-1}\left(J_{\bar{u}_{n}} \cap\left(\bar{B}_{r^{\prime \prime}} \backslash B_{r^{\prime}}\right)\right)+\tilde{D}_{n}\left(r^{\prime \prime}\right) \\
\leq & \int_{B_{r^{\prime}}} f_{n}(x, \nabla v) d x+\int_{B_{r^{\prime \prime}} \backslash B_{r^{\prime}}} \tilde{L}\left(1+|\nabla v|^{p}+\left|\nabla \bar{u}_{n}\right|^{p}+|\nabla \varphi|^{p}\left|v-\bar{u}_{n}\right|^{p}\right) d x \\
& +\Lambda c_{n} \mathcal{H}^{d-1}\left(J_{\bar{u}_{n}} \cap\left(\bar{B}_{r^{\prime \prime}} \backslash B_{r^{\prime}}\right)\right)+\tilde{D}_{n}\left(r^{\prime \prime}\right),
\end{aligned}
$$

where $\tilde{L}>0$ is independent of $n$. Letting $n \rightarrow+\infty$ we get using the uniform convergence of $f_{n}$ to $f_{\infty}$ and (4.17)

$$
\begin{aligned}
& \liminf _{n \rightarrow+\infty}\left[\int_{B_{r^{\prime \prime}}} f_{n}\left(x, \nabla \bar{u}_{n}\right) d x+c_{n} \mathcal{H}^{d-1}\left(J_{\bar{u}_{n}} \cap \bar{B}_{r^{\prime \prime}}\right)\right] \\
& \quad \leq \int_{B_{r^{\prime}}} f_{\infty}(x, \nabla v) d x+\tilde{L} \int_{B_{r^{\prime \prime}} \backslash B_{r^{\prime}}}\left(1+|\nabla v|^{p}\right) d x+\Lambda \mu\left(\bar{B}_{r^{\prime \prime}} \backslash B_{r^{\prime}}\right) .
\end{aligned}
$$

Letting $r^{\prime \prime} \rightarrow r^{\prime}$, and since $\mu\left(\partial B_{r^{\prime}}\right)=0$ we deduce

$$
\alpha\left(r^{\prime}\right) \leq \int_{B_{r^{\prime}}} f_{\infty}(x, \nabla v) d x
$$

Moreover, thanks to (4.16), for $r^{\prime} \rightarrow r$ we obtain

$$
\int_{B_{r}} f_{\infty}(x, \nabla u) d x \leq \alpha(r) \leq \int_{B_{r}} f_{\infty}(x, \nabla v) d x .
$$

We conclude that $u$ is a local minimizer in $W^{1, p}\left(B_{1}\right)$ for

$$
v \mapsto \int_{B_{1}} f_{\infty}(x, \nabla v) d x
$$

and that for every $r \in(0,1)$ (take $v=u$ )

$$
\alpha(r)=\int_{B_{r}} f_{\infty}(x, \nabla u) d x .
$$

We note that the previous equality together with (4.15) entails that for every $r \in(0,1)$

$$
\lim _{n \rightarrow+\infty} \int_{B_{r}} f_{n}\left(x, \nabla \bar{u}_{n}\right) d x=\int_{B_{r}} f_{\infty}(x, \nabla u) d x \quad \text { and } \quad \lim _{n \rightarrow+\infty} c_{n} \mathcal{H}^{d-1}\left(J_{\bar{u}_{n}} \cap \bar{B}_{r}\right)=0 .
$$

In order to obtain (4.11), we need to replace $\bar{u}_{n}$ with $u_{n}$. Let $\tilde{r}>r$ be such that according to relation (4.12)

$$
c_{n} \mathcal{H}^{d-1}\left(\left\{\bar{u}_{n} \neq u_{n}\right\} \cap \partial B_{\tilde{r}}\right) \rightarrow 0 .
$$

Comparing $u_{n}$ with $\bar{u}_{n} 1_{B_{\tilde{r}}}+u_{n} 1_{B_{1} \backslash B_{\tilde{r}}}$ we get

$$
\begin{aligned}
& \int_{B_{\tilde{r}}} f_{n}\left(x, \nabla u_{n}\right) d x+c_{n} \mathcal{H}^{d-1}\left(J_{u_{n}} \cap \bar{B}_{\tilde{r}}\right) \\
& \quad \leq \int_{B_{\tilde{r}}} f_{n}\left(x, \nabla \bar{u}_{n}\right) d x+\Lambda c_{n} \mathcal{H}^{d-1}\left(J_{\bar{u}_{n}} \cap B_{\tilde{r}}\right)+\Lambda c_{n} \mathcal{H}^{d-1}\left(\left\{\bar{u}_{n} \neq u_{n}\right\} \cap \partial B_{\tilde{r}}\right)+D_{n}(\tilde{r})
\end{aligned}
$$


so that recalling (4.16), (4.18) and (4.19)

$$
\begin{aligned}
\int_{B_{r}} f_{\infty}(x, \nabla u) d x & \leq \liminf _{n \rightarrow+\infty}\left[\int_{B_{r}} f_{n}\left(x, \nabla u_{n}\right) d x+c_{n} \mathcal{H}^{d-1}\left(J_{u_{n}} \cap \bar{B}_{r}\right)\right] \\
& \leq \limsup _{n \rightarrow+\infty}\left[\int_{B_{r}} f_{n}\left(x, \nabla u_{n}\right) d x+c_{n} \mathcal{H}^{d-1}\left(J_{u_{n}} \cap \bar{B}_{r}\right)\right] \leq \int_{B_{\tilde{r}}} f_{\infty}(x, \nabla u) d x,
\end{aligned}
$$

from which (4.11) follows letting $\tilde{r} \rightarrow r$.

We need now to adapt the previous lemma to the case with boundary conditions. Let $\varphi_{n}: \mathbb{R}^{d-1} \rightarrow \mathbb{R}$ be a sequence of continuous functions, and let

$$
T_{n}:=\left\{x=\left(x^{\prime}, x_{d}\right) \in B_{1}: x_{d} \leq \varphi_{n}\left(x^{\prime}\right)\right\} .
$$

Assume that $\left(\varphi_{n}\right)_{n \in \mathbb{N}}$ is locally uniformly converging to the constant function $-\delta$, with $\delta \in[0,1)$. Let $\left(g_{n}\right)_{n \in \mathbb{N}}$ be a sequence in $C^{1}\left(\bar{B}_{1}\right)$ such that

$$
\left\|g_{n}\right\|_{\infty}+\left\|\nabla g_{n}\right\|_{\infty} \rightarrow 0 .
$$

Let $\left(u_{n}\right)_{n \in \mathbb{N}}$ be a sequence in $S B V\left(B_{1}\right)$ with $u_{n}=g_{n}$ on $T_{n}$, which satisfy the minimality property

$$
\begin{aligned}
& \int_{B_{r}} f_{n}\left(x, \nabla u_{n}\right) d x+c_{n} \mathcal{H}^{d-1}\left(J_{u_{n}} \cap \bar{B}_{r}\right) \\
& \quad \leq \int_{B_{r}} f_{n}(x, \nabla v) d x+\Lambda c_{n} \mathcal{H}^{d-1}\left(J_{v} \cap \bar{B}_{r}\right)+D_{n}(r)
\end{aligned}
$$

for every $v \in S B V\left(B_{1}\right)$ with $v=g_{n}$ on $T_{n},\left\{v \neq u_{n}\right\} \subseteq B_{r}, r \in[0,1]$, and with $D_{n}$ satisfying (4.5). Let finally $F_{0, \delta}^{\infty}$ : $W^{1, p}\left(B_{1}\right) \rightarrow[0,+\infty[$ be given by

$$
F_{0, \delta}^{\infty}(u):= \begin{cases}\int_{B_{1}} f_{\infty}(x, \nabla u) & \text { if } u=0 \text { on }\left\{x \in B_{1}: x_{d}<-\delta\right\} \\ +\infty & \text { otherwise. }\end{cases}
$$

We say that $u$ is a local minimizer of $F_{0, \delta}^{\infty}$ if for every $v \in W^{1, p}\left(B_{1}\right)$ with $v=0$ on $\left\{x \in B_{1}: x_{d}<-\delta\right\}$ and $\{v \neq$ $u\} \subset \subset B_{1}$ we have $F_{0, \delta}^{\infty}(u) \leq F_{0, \delta}^{\infty}(v)$.

The following lemma holds true.

Lemma 4.7. Let $\left(u_{n}\right)_{n \in \mathbb{N}}$ be a sequence in $S B V\left(B_{1}\right)$ with $u_{n}=g_{n}$ on $T_{n}$, and satisfying the almost-quasi minimality property (4.21). Assume that

$$
\begin{aligned}
& \sup _{n} \int_{B_{1}}\left|\nabla u_{n}\right|^{p} d x<+\infty, \quad \mathcal{H}^{d-1}\left(J_{u_{n}}\right) \rightarrow 0, \\
& \sup _{n}\left[\int_{B_{1}} f_{n}\left(x, \nabla u_{n}\right) d x+c_{n} \mathcal{H}^{d-1}\left(J_{u_{n}}\right)\right]<+\infty
\end{aligned}
$$

and

$$
u_{n} \rightarrow u \in W^{1, p}\left(B_{1}\right) \quad \text { pointwise a.e. }
$$

Then $u$ is a local minimizer in $W^{1, p}\left(B_{1}\right)$ of the functional $F_{0, \delta}^{\infty}$ and for every $r \in[0,1]$

$$
\lim _{n \rightarrow+\infty} \int_{B_{r}} f_{n}\left(x, \nabla u_{n}\right) d x=\int_{B_{r}} f_{\infty}(x, \nabla u) d x \quad \text { and } \quad \lim _{n \rightarrow+\infty} c_{n} \mathcal{H}^{d-1}\left(J_{u_{n}} \cap \bar{B}_{r}\right)=0 .
$$


Proof. If we set $v_{n}:=u_{n}-g_{n}$, we get easily that for $n$ large enough

$$
\bar{v}_{n}=0 \quad \text { on } T_{n} .
$$

Setting

$$
\bar{u}_{n}:=g_{n}+\bar{v}_{n}
$$

we have

$$
\bar{u}_{n} \rightarrow u \quad \text { strongly in } L^{p}\left(B_{1}\right)
$$

and

$$
\left(1+c_{n}\right)\left|\left\{\bar{u}_{n} \neq u_{n}\right\}\right| \rightarrow 0 .
$$

We can then follow the proofs of lemma and of Lemma 4.6 to get the conclusion: it suffices to note that every $v \in W^{1, p}\left(B_{1}\right)$ with $v=0$ on $\left\{x \in B_{1}: x_{d} \leq-\delta\right\}$, in view of (4.20), there exists $v_{n} \in W^{1, p}\left(B_{1}\right)$ with $v_{n}=g_{n}$ on $T_{n}$ and such that $v_{n} \rightarrow v$ strongly in $W^{1, p}\left(B_{1}\right)$.

\subsection{The decay lemma}

For every ball $B_{\rho}\left(x_{0}\right) \subset \subset \Omega^{\prime}$ let us write

$$
F\left(u, B_{\rho}\left(x_{0}\right)\right):=\int_{B_{\rho}} f(x, \nabla u) d x+\mathcal{H}^{d-1}\left(J_{u} \cap B_{\rho}\right) .
$$

Let us denote by $\operatorname{Dev}_{g, \Lambda}\left(u, B_{\rho}\left(x_{0}\right)\right)$ the infimum of those constants $D$ such that

$$
\int_{B_{\rho}\left(x_{0}\right)} f(x, \nabla u) d x+\mathcal{H}^{d-1}\left(J_{u} \cap \bar{B}_{\rho}\left(x_{0}\right)\right) \leq \int_{B_{\rho}\left(x_{0}\right)} f(x, \nabla v) d x+\Lambda \mathcal{H}^{d-1}\left(J_{v} \cap \bar{B}_{\rho}\left(x_{0}\right)\right)+D
$$

for every $v \in S B V^{p}\left(\Omega^{\prime}\right)$ with $v=g$ on $\Omega^{\prime} \backslash \bar{\Omega}$ and such that $\{v \neq u\} \subseteq B_{\rho}\left(x_{0}\right)$.

The decay lemma with boundary conditions which we will use is the following.

Lemma 4.8 (Decay). Assume that $f$ satisfies assumptions $\left(H_{1}\right)-\left(H_{3}\right)$. For every $\tau \in(0,1)$ and $\tilde{\Omega} \subset \subset \Omega^{\prime}$ there exist $\varepsilon(\tau, \tilde{\Omega}), \vartheta(\tau, \tilde{\Omega}), \rho(\tau, \tilde{\Omega}), \chi(\tau, \tilde{\Omega})>0$ such that if $u \in S B V^{p}\left(\Omega^{\prime}\right)$ with $u=g$ on $\Omega^{\prime} \backslash \bar{\Omega}, x \in \tilde{\Omega}, \rho<\rho(\tau, \tilde{\Omega})$, $B_{\rho}(x) \subset \subset \Omega^{\prime}$,

$$
\mathcal{H}^{d-1}\left(J_{u} \cap B_{\rho}(x)\right) \leq \varepsilon(\tau, \tilde{\Omega}) \rho^{d-1} \quad \text { and } \quad \operatorname{Dev}_{g, \Lambda}\left(u, B_{\rho}(x)\right) \leq \vartheta(\tau, \tilde{\Omega}) F\left(u, B_{\rho}(x)\right)
$$

then

$$
F\left(u, B_{\tau \rho}(x)\right) \leq C_{1} \tau^{d} \max \left\{F\left(u, B_{\rho}(x)\right), \chi(\tau, \tilde{\Omega}) \rho^{d}\left[\operatorname{Lip}_{B_{\rho}(x)} g\right]^{p}\right\},
$$

where $C_{1}>0$ depends only on the dimension $d$ and on the constants $L, \mu, p$ associated to $f$.

Proof. It suffices to consider the case $\tau \in(0,1 / 4)$ (otherwise we can choose $C_{1}=4^{d}$ ). Let $C_{1}>0$ to be fixed below. By contradiction, let us assume that there exist

$$
\tau \in(0,1), \quad \varepsilon_{n} \rightarrow 0, \quad \vartheta_{n} \rightarrow 0, \quad \rho_{n} \rightarrow 0, \quad \chi_{n} \rightarrow+\infty, \quad x_{n} \in \tilde{\Omega}
$$

such that $B_{\rho_{n}}\left(x_{n}\right) \subset \subset \Omega^{\prime}$,

$$
\mathcal{H}^{d-1}\left(J_{u} \cap B_{\rho_{n}}\left(x_{n}\right)\right)=\varepsilon_{n} \rho_{n}^{d-1}, \quad \operatorname{Dev}_{g, \Lambda}\left(u, B_{\rho_{n}}\left(x_{n}\right)\right)=\vartheta_{n} F\left(u, B_{\rho_{n}}\left(x_{n}\right)\right),
$$

and

$$
F\left(u, B_{\tau \rho_{n}}\left(x_{n}\right)\right)>C_{1} \tau^{d} \max \left\{F\left(u, B_{\rho_{n}}\left(x_{n}\right)\right), \chi_{n} \rho_{n}^{d}\left[\operatorname{Lip}_{B_{\rho_{n}}\left(x_{n}\right)} g\right]^{p}\right\} .
$$


We consider the rescaled function $v_{n} \in S B V^{p}\left(B_{1}\right)$ given by

$$
v_{n}(y):=\rho_{n}^{\frac{1-p}{p}} c_{n}^{\frac{1}{p}} u\left(x_{n}+\rho_{n} y\right)
$$

where

$$
c_{n}:=\frac{\rho_{n}^{d-1}}{F\left(u_{n}, B_{\rho_{n}}\left(x_{n}\right)\right)} .
$$

We thus get

$$
\int_{B_{1}} f_{n}\left(y, \nabla v_{n}(y)\right) d y+c_{n} \mathcal{H}^{d-1}\left(J_{v_{n}}\right)=1,
$$

where

$$
f_{n}(y, \xi):=f\left(x_{n}+\rho_{n} y, \xi\right),
$$

and

$$
\sup _{n} \int_{B_{1}}\left|\nabla v_{n}\right|^{p} d y<+\infty \quad \text { and } \quad \mathcal{H}^{d-1}\left(J_{v_{n}}\right) \rightarrow 0 .
$$

If we define $g_{n}$ starting from $g$ as in (4.24), we get

$$
\int_{B_{\tau}} f_{n}\left(y, \nabla v_{n}(y)\right) d y+c_{n} \mathcal{H}^{d-1}\left(J_{v_{n}} \cap \bar{B}_{\tau}\right)>C_{1} \tau^{d} \max \left\{1, \chi_{n}\left[\operatorname{Lip}_{B_{1}} g_{n}\right]^{p}\right\} \geq C_{1} \tau^{d},
$$

so that in particular

$$
\operatorname{Lip}_{B_{1}} g_{n} \leq \frac{1}{\left[C_{1} \tau^{d} \chi_{n}\right]^{1 / p}} \rightarrow 0 .
$$

Finally $v_{n}$ satisfies the minimality property (4.21), with respect to the region $T_{n}$ corresponding to $\left(\Omega^{\prime} \backslash \bar{\Omega}\right) \cap B_{\rho_{n}}\left(x_{n}\right)$, the given datum $g_{n}$, and $D_{n}(r)=\vartheta_{n}$ for every $r \in[0,1]$.

Up to a subsequence, we may assume that $x_{n} \rightarrow x_{\infty} \in \bar{\Omega}$, so that

$$
f_{n}(y, \xi) \rightarrow f\left(x_{\infty}, \xi\right) \quad \text { uniformly on compact subsets of } B_{1} \times \mathbb{R}^{d} .
$$

It is not restrictive to assume $x_{\infty} \in \bar{\Omega}$ (otherwise for $n$ large we have $u=g$ on $B_{\rho_{n}}\left(x_{n}\right)$, and the result is trivial). We now divide three cases.

Case 1 Assume that $T_{n}=\emptyset$ for $n$ large. Thanks to Lemma 4.4 and Lemma 4.6, and since the energy is invariant under the addition of a constant, we may assume that

$$
v_{n} \rightarrow v \in W^{1, p}\left(B_{1}\right) \quad \text { pointwise a.e. }
$$

with $v$ local minimizer of $w \mapsto \int_{B_{1}} f\left(x_{\infty}, \nabla w(y)\right) d y$ on $W^{1, p}\left(B_{1}\right)$, and such that every $r \in[0,1]$

$$
\lim _{n \rightarrow+\infty} \int_{B_{r}} f_{n}\left(y, \nabla v_{n}(y)\right) d y=\int_{B_{r}} f\left(x_{\infty}, \nabla v(y)\right) d y \quad \text { and } \quad \lim _{n \rightarrow+\infty} c_{n} \mathcal{H}^{d-1}\left(J_{v_{n}} \cap \bar{B}_{r}\right)=0 .
$$

In particular

$$
\int_{B_{1}} f\left(x_{\infty}, \nabla v(y)\right) d y=1 .
$$


However, by Theorem $4.2 v$ is Lipschitz continuous with

$$
\sup _{B_{1 / 2}}|\nabla v|^{p} \leq \frac{C_{0}}{\omega_{d}} \int_{B_{1}} f\left(x_{\infty}, \nabla v\right) d y \leq \frac{C_{0}}{\omega_{d}},
$$

where $C_{0}=C_{0}(d, p, L, \mu)$. Hence we get

$$
\int_{B_{\tau}} f\left(x_{\infty}, \nabla v(y)\right) d y \leq L \int_{B_{\tau}}|\nabla v|^{p} d y \leq L \omega_{d} \tau^{d} \sup _{B_{1 / 4}}|\nabla v|^{p} \leq L C_{0} \tau^{d} .
$$

We get a contradiction with (4.27) if we choose $C_{1}>L C_{0}$.

Case 2 Assume that $T_{n} \neq \emptyset$ for $n$ large. Since $\rho_{n} \rightarrow 0$, we get $x_{\infty} \in \partial\left(\Omega^{\prime} \backslash \bar{\Omega}\right)$. Since $\Omega^{\prime} \backslash \bar{\Omega}$ has $C^{1}$-boundary, there exists a coordinate system such that, up to a subsequence,

$$
T_{n}=\left\{y=\left(y^{\prime}, y_{d}\right) \in B_{1}: y_{d} \leq \varphi_{n}\left(y^{\prime}\right)\right\}
$$

for some $\varphi_{n} \in C^{1}\left(\mathbb{R}^{d-1}\right)$ locally uniformly converging to a constant $-\delta$, with $\delta \in[0,1]$ (see e.g. [3, Lemma 6.4]).

Thanks to (4.26) and to Lemma 4.4, there exists $v \in W^{1, p}\left(B_{1}\right)$ such that up to a subsequence (not relabeled)

$$
v_{n}-m_{n} \rightarrow v \quad \text { a.e. in } B_{1},
$$

where $m_{n}$ denotes a median of $v_{n}$.

Assume now that $0 \leq \delta \leq \frac{1}{2}$. In view of (4.28), we conclude that $v$ is constant on $\left\{y=\left(y^{\prime}, y_{d}\right) \in B_{1}: y_{d} \leq-\delta\right\}$. It is not restrictive (since the energies are invariant by addition of a constant) to assume that $m_{n}=0$,

$$
v=0 \quad \text { on }\left\{y=\left(y^{\prime}, y_{d}\right) \in B_{1}: y_{d} \leq-\delta\right\}
$$

and that

$$
v_{n} \rightarrow v \quad \text { a.e. in } B_{1} .
$$

By Lemma 4.7 we deduce that $v$ is a local minimizer of the functional $F_{0, \delta}^{\infty}$ associated to the energy density $f\left(x_{\infty}, \xi\right)$,

$$
\lim _{n \rightarrow+\infty} \int_{B_{\tau}} f_{n}\left(y, \nabla v_{n}(y)\right) d y=\int_{B_{\tau}} f\left(x_{\infty}, \nabla v\right) d y, \quad \lim _{n \rightarrow+\infty} c_{n} \mathcal{H}^{d-1}\left(J_{v_{n}} \cap \bar{B}_{\tau}\right)=0
$$

and

$$
\int_{B_{1}} f\left(x_{\infty}, \nabla v\right) d y=1
$$

In view of Theorem 4.3, $v$ is locally Lipschitz in $B_{1}$, and there exists a constant $C_{0}^{\prime}=C_{0}^{\prime}(d, p, L, \mu)$ such that

$$
\sup _{B_{\tau}}|\nabla v|^{p} \leq C_{0}^{\prime}\left[\int_{B_{1}}|\nabla v|^{p} d x+1\right] .
$$

Consequently we have

$$
\int_{B_{\tau}} f\left(x_{\infty}, \nabla v\right) d y \leq L C_{0}^{\prime}\left[\int_{B_{1}}|\nabla v|^{p} d x+1\right] \omega_{d} \tau^{d}
$$

and we get a contradiction with (4.27) if

$$
C_{1}>L(L+1) C_{0}^{\prime} \omega_{d} .
$$

Let now $\frac{1}{2}<\delta \leq 1$. Then for $n$ large enough

$$
T_{n} \cap B_{1 / 2}=\emptyset \text {. }
$$


By Lemma 4.6 we infer that $v$ is a local minimizer of $F_{\infty}$ associated to $f\left(x_{\infty}, \xi\right)$ on $B_{1 / 2}$,

$$
\lim _{n \rightarrow+\infty} \int_{B_{r}} f_{n}\left(y, \nabla v_{n}(y)\right) d y=\int_{B_{r}} f\left(x_{\infty}, \nabla v\right) d x, \quad \lim _{n \rightarrow+\infty} \mathcal{H}^{d-1}\left(J_{v_{n}} \cap \bar{B}_{r}\right)=0
$$

for every $0 \leq r \leq 1 / 2$ and

$$
\int_{B_{1 / 2}} f\left(x_{\infty}, \nabla v\right) d x \leq 1 .
$$

Since $v$ is Lipschitz continuous thanks to Theorem 4.2 with

$$
\sup _{B_{1 / 4}}|\nabla v|^{p} \leq \frac{\tilde{C}_{0}}{\omega_{d}} \int_{B_{1 / 2}} f\left(x_{\infty}, \nabla v\right) d y \leq \frac{\tilde{C}_{0}}{\omega_{d}}
$$

where $\tilde{C}_{0}=\tilde{C}_{0}(d, p, L, \mu)$, we get as $\tau \leq 1 / 4$

$$
\int_{B_{\tau}} f\left(x_{\infty}, \nabla v(y)\right) d y \leq L \int_{B_{\tau}}|\nabla v|^{p} d y \leq L \omega_{d} \tau^{d} \sup _{B_{1 / 4}}|\nabla v|^{p} \leq L \tilde{C}_{0} \tau^{d} .
$$

We get a contradiction with (4.27) if we choose $C_{1}>L \tilde{C}_{0}$. The proof is now concluded.

\subsection{Proof of Theorem 2.3}

By comparing $u$ with $u 1_{B_{\rho}(x)}$ for $B_{\rho}(x) \subset \subset \Omega^{\prime}$, we get easily that

$$
\mathcal{H}^{d-1}\left(J_{u} \cap B_{\rho}(x)\right) \leq \Lambda \omega_{d} \rho^{d-1}+c_{\alpha} \rho^{d-1+\alpha} \leq\left(\Lambda \omega_{d}+1\right) \rho^{d-1}
$$

for $\rho$ small enough (depending only on $\alpha$ and $c_{\alpha}$ ). Let $\tilde{\Omega} \subset \subset \Omega^{\prime}$ be fixed. We claim that we can find $\varepsilon_{0}, \rho_{0}>0$ depending on $\tilde{\Omega}$ such that the relations

$$
x \in \tilde{\Omega}, \quad B_{\rho}(x) \subset \subset \Omega^{\prime} \quad \text { and } \quad \mathcal{H}^{d-1}\left(J_{u} \cap B_{\rho}(x)\right) \leq \varepsilon_{0} \rho^{d-1} \text { for some } \rho<\rho_{0}
$$

entails

$$
\lim _{r \rightarrow 0} \frac{\int_{B_{r}(x)}|\nabla u|^{p} d x+\mathcal{H}^{d-1}\left(J_{u} \cap B_{r}(x)\right)}{r^{d-1}}=0 .
$$

Then the thesis follows since thanks to [10, Theorem 3.6] relation (4.29) implies $x \notin J_{u}$. We can thus achieve inequality (2.2) by reducing $\varepsilon_{0}$ if necessary.

In order to prove (4.29), it is not restrictive to assume $\rho_{0}<1$. We will use the almost-quasi minimality property of Definition 2.2 only on balls with radius $\rho<\rho_{0}$, so that we can assume without loss of generality that $\alpha \leq 1$.

We proceed as follows. Let $\tau \in(0,1)$ be such that

$$
C_{1} \tau^{d} \leq \tau^{d-\frac{\alpha}{2}}
$$

where $C_{1}$ is the constant given by the Decay Lemma 4.8. Let $\sigma \in(0,1)$ to be fixed below. We claim that there exists $\rho_{0}>0$ such that if for some $\rho<\rho_{0}$

$$
\mathcal{H}^{d-1}\left(J_{u} \cap B_{\rho}(x)\right) \leq \varepsilon(\sigma) \rho^{d-1}
$$

then

$$
F\left(u, B_{\sigma \tau^{h} \rho}\right) \leq \varepsilon(\tau) \tau^{\frac{\alpha h}{2}}\left(\sigma \tau^{h} \rho\right)^{d-1} .
$$

Here $\varepsilon(\tau)$ and $\varepsilon(\sigma)$ are the numbers associated to $\tau, \sigma$ and $\tilde{\Omega}$ according to the Decay Lemma 4.8. From this inequality, relation (4.29) easily follows, and the thesis is proved by choosing $\varepsilon_{0}=\varepsilon(\sigma)$. 
We prove (4.31) by induction. In what follows, $L_{g}$ will denote the Lipschitz constant of $g$ on $\tilde{\Omega}$. For $h=0$ it reads

$$
F\left(u, B_{\sigma \rho}\right) \leq \varepsilon(\tau)(\sigma \rho)^{d-1} .
$$

If

$$
\operatorname{Dev}_{g, \Lambda}\left(u, B_{\rho}(x)\right) \leq \vartheta(\sigma) F\left(u, B_{\rho}(x)\right),
$$

we get according to Lemma 4.8, and using the almost-quasi minimality property of $u$ in comparison with $g 1_{B_{\rho}(x)}+$ $u 1_{\Omega^{\prime} \backslash B_{\rho}(x)}$

$$
\begin{aligned}
F\left(u, B_{\sigma \rho}(x)\right) & \leq C_{1} \sigma^{d} \max \left\{F\left(u, B_{\rho}(x)\right), \chi(\sigma) \rho^{d}\left[\operatorname{Lip}_{B_{\rho}(x)} g\right]^{p}\right\} \\
& \leq C_{1} \sigma^{d} \max \left\{\omega_{d} \rho^{d} L\left[\operatorname{Lip}_{B_{\rho}(x)} g\right]^{p}+d \omega_{d} \rho^{d-1}+c_{\alpha} \rho^{d-1+\alpha}, \chi(\sigma) \rho^{d}\left[\operatorname{Lip}_{B_{\rho}(x)} g\right]^{p}\right\} \\
& \leq(\rho \sigma)^{d-1} C_{1} \sigma \max \left\{\omega_{d} \rho L L_{g}^{p}+d \omega_{d}+c_{\alpha} \rho^{\alpha}, \chi(\sigma) \rho L_{g}^{p}\right\} \leq \varepsilon(\tau)(\sigma \rho)^{d-1}
\end{aligned}
$$

provided that

$$
\rho_{0} \leq \min \left\{\frac{1}{\chi(\sigma)}, 1\right\}
$$

and

$$
C_{1} \sigma \max \left\{\omega_{d} L L_{g}^{p}+d \omega_{d}+c_{\alpha}, L_{g}^{p}\right\} \leq \varepsilon(\tau) .
$$

If on the other hand

$$
\operatorname{Dev}_{g, \Lambda}\left(u, B_{\rho}(x)\right)>\vartheta(\sigma) F\left(u, B_{\rho}(x)\right)
$$

then by the minimality property of $u$

$$
F\left(u, B_{\sigma \rho}(x)\right) \leq F\left(u, B_{\rho}(x)\right) \leq \frac{1}{\vartheta(\sigma)} \operatorname{Dev}_{g, \Lambda}\left(u, B_{\rho}\right) \leq \frac{c_{\alpha} \rho^{d-1+\alpha}}{\vartheta(\sigma)} \leq \varepsilon(\tau)(\sigma \rho)^{d-1}
$$

provided that

$$
c_{\alpha} \rho_{0}^{\alpha}<\varepsilon(\tau) \vartheta(\sigma) \sigma^{d-1} .
$$

Relation (4.32) is now completely proved.

Assume now that (4.31) holds true. We want to see that

$$
F\left(u, B_{\sigma \tau^{h+1} \rho}\right) \leq \varepsilon(\tau) \tau^{\frac{\alpha(h+1)}{2}}\left(\sigma \tau^{h+1} \rho\right)^{d-1} .
$$

From (4.31) we infer that

$$
\mathcal{H}^{d-1}\left(J_{u} \cap B_{\sigma \tau^{h} \rho}\right) \leq \varepsilon(\tau)\left(\sigma \tau^{h} \rho\right)^{d-1} .
$$

If

$$
\operatorname{Dev}_{g, \Lambda}\left(u, B_{\sigma \tau^{h} \rho}(x)\right) \leq \vartheta(\tau) F\left(u, B_{\sigma \tau^{h} \rho}\right)
$$

then by the Decay Lemma 4.8

$$
\begin{aligned}
F\left(u, B_{\sigma \tau^{h+1} \rho}\right) & \leq C_{1} \tau^{d} \max \left\{F\left(u, B_{\sigma \tau^{h} \rho}\right), \chi(\tau)\left(\sigma \tau^{h} \rho\right)^{d} L_{g}^{p}\right\} \\
& \leq C_{1} \tau^{d} \max \left\{\varepsilon(\tau) \tau^{\frac{\alpha h}{2}}\left(\sigma \tau^{h} \rho\right)^{d-1}, \chi(\tau)\left(\sigma \tau^{h} \rho\right)^{d} L_{g}^{p}\right\} \\
& =C_{1} \tau^{d}\left(\sigma \tau^{h} \rho\right)^{d-1} \tau^{\frac{\alpha h}{2}} \max \left\{\varepsilon(\tau), \chi(\tau) \sigma \tau^{\frac{(2-\alpha) h}{2}} \rho L_{g}^{p}\right\} .
\end{aligned}
$$

If

$$
\chi(\tau) \rho_{0} L_{g}^{p} \leq \varepsilon(\tau),
$$


and since $\alpha \leq 1$, we infer taking into account also (4.30)

$$
\begin{aligned}
F\left(u, B_{\sigma \tau^{h+1} \rho}\right) & \leq \varepsilon(\tau) \tau^{d-\frac{\alpha}{2}}\left(\sigma \tau^{h} \rho\right)^{d-1} \tau^{\frac{\alpha h}{2}}=\varepsilon(\tau) \tau^{1-\alpha} \tau^{\frac{\alpha(h+1)}{2}}\left(\sigma \tau^{h+1} \rho\right)^{d-1} \\
& \leq \varepsilon(\tau) \tau^{\frac{\alpha(h+1)}{2}}\left(\sigma \tau^{h+1} \rho\right)^{d-1} .
\end{aligned}
$$

If on the contrary

$$
\operatorname{Dev}_{g, \Lambda}\left(u, B_{\sigma \tau^{h} \rho}(x)\right)>\vartheta(\tau) F\left(u, B_{\sigma \tau^{h} \rho}\right)
$$

we have using the minimality property of $u$

$$
\begin{aligned}
F\left(u, B_{\sigma \tau^{h+1} \rho}(x)\right) & \leq F\left(u, B_{\sigma \tau^{h} \rho}(x)\right) \leq \frac{1}{\vartheta(\tau)} \operatorname{Dev}_{g, \Lambda}\left(u, B_{\sigma \tau^{h} \rho}(x)\right) \leq \frac{1}{\vartheta(\tau)} c_{\alpha}\left(\sigma \tau^{h} \rho\right)^{d-1+\alpha} \\
& =\frac{c_{\alpha}\left(\sigma \tau^{h} \rho\right)^{\alpha}}{\vartheta(\tau)} \frac{\left(\sigma \tau^{h+1} \rho\right)^{d-1}}{\tau^{d-1}} \leq \varepsilon(\tau) \tau^{\frac{\alpha(h+1)}{2}}\left(\sigma \tau^{h+1} \rho\right)^{d-1}
\end{aligned}
$$

provided that

$$
c_{\alpha} \rho_{0}^{\alpha} \leq \varepsilon(\tau) \vartheta(\tau) \tau^{d-1+\frac{\alpha}{2}} .
$$

Relation (4.36) thus follows.

Summarizing, we conclude that (4.31) holds true provided that $\sigma$ satisfies (4.34) and $\rho_{0}$ satisfies (4.33), (4.35), and (4.38). The proof is now concluded.

\section{Conflict of interest statement}

We confirm that we do not have any conflict.

\section{References}

[1] H.W. Alt, L.A. Caffarelli, Existence and regularity for a minimum problem with free boundary, J. Reine Angew. Math. 325 (1981) $105-144$.

[2] L. Ambrosio, N. Fusco, D. Pallara, Functions of Bounded Variation and Free Discontinuity Problems, Oxf. Math. Monogr., The Clarendon Press, Oxford University Press, New York, 2000.

[3] J.-F. Babadjian, A. Giacomini, Existence of strong solutions for quasi-static evolution in brittle fracture, Ann. Sc. Norm. Super. Pisa Cl. Sci. (5) XIII (2014) 925-974.

[4] A. Braides, Approximation of Free-Discontinuity Problems, Lect. Notes Math., vol. 1694, Springer-Verlag, Berlin, 1998.

[5] D. Bucur, A. Giacomini, Faber-Krahn inequalities for the Robin-Laplacian: a free discontinuity approach, Arch. Ration. Mech. Anal. 218 (2) (2015) 757-824.

[6] D. Bucur, S. Luckhaus, Monotonicity formula and regularity for general free discontinuity problems, Arch. Ration. Mech. Anal. 211 (2) (2014) 489-511.

[7] M. Carriero, A. Leaci, Existence theorem for a Dirichlet problem with free discontinuity set, Nonlinear Anal. 15 (7) (1990) $661-677$.

[8] G. Cortesani, R. Toader, A density result in SBV with respect to non-isotropic energies, Nonlinear Anal., Ser. B: Real World Appl. 38 (5) (1999) 585-604.

[9] E. De Giorgi, L. Ambrosio, New functionals in the calculus of variations, Atti Accad. Naz. Lincei, Rend. Cl. Sci. Fis. Mat. Natur. (8) 82 (2) (1988) 199-210 (1989).

[10] E. De Giorgi, M. Carriero, A. Leaci, Existence theorem for a minimum problem with free discontinuity set, Arch. Ration. Mech. Anal. 108 (3) (1989) 195-218.

[11] I. Fonseca, N. Fusco, Regularity results for anisotropic image segmentation models, Ann. Sc. Norm. Super. Pisa, Cl. Sci. (4) 24 (3) (1997) 463-499.

[12] N. Fusco, G. Mingione, C. Trombetti, Regularity of minimizers for a class of anisotropic free discontinuity problems, J. Convex Anal. 8 (2) (2001) 349-367.

[13] A. Siaudeau, Ahlfors-régularité des quasi-minima de Mumford-Shah, J. Math. Pures Appl. (9) 82 (12) (2003) $1697-1731$. 